\title{
Triple-negative breast cancer therapy: Current and future perspectives (Review)
}

\author{
KWANG-AI WON ${ }^{1,2}$ and CHARLES SPRUCK ${ }^{3}$ \\ ${ }^{1}$ ConsultantCA, Moraga, CA 94556; ${ }^{2}$ Pin Pharmaceuticals, Inc., South San Francisco, CA 94080; \\ ${ }^{3}$ Tumor Initiation and Maintenance Program, NCI-Designated Cancer Center, \\ Sanford Burnham Prebys Medical Discovery Institute, La Jolla, CA 92037, USA
}

Received July 14, 2020; Accepted September 9, 2020

DOI: $10.3892 /$ ijo.2020.5135

\begin{abstract}
Triple-negative breast cancer (TNBC) accounts for $10-15 \%$ of all breast cancer cases. TNBCs lack estrogen and progesterone receptors and express low levels of HER2, and therefore do not respond to hormonal or anti-HER2 therapies. TNBC is a particularly aggressive form of breast cancer that generally displays poorer prognosis compared to other breast cancer subtypes. TNBC is chemotherapy sensitive, and this treatment remains the standard of care despite its limited benefit. Recent advances with novel agents have been made for specific subgroups with PD-L1 ${ }^{+}$tumors or germline Brca-mutated tumors. However, only a fraction of these patients responds to immune checkpoint or PARP inhibitors and even those who do respond often develop resistance and relapse. Various new agents and combination strategies have been explored to further understand molecular and immunological aspects of TNBC. In this review, we discuss clinical trials in the management of TNBC as well as perspectives for potential future treatments.
\end{abstract}

\section{Contents}

1. Introduction

2. Current treatment paradigm

3. Investigational drugs

4. New potential therapeutic strategies

5. Conclusion

\section{Introduction}

Breast cancer is characterized by heterogeneity at the molecular and clinical levels. Several biomarkers including

Correspondence to: Dr Kwang-Ai Won, ConsultantCA, 1988 Ascot Drive, Unit A, Moraga, CA 94556, USA

E-mail: wonk12pharm@yahoo.com

Key words: triple-negative breast cancer, clinical studies, immunotherapy, DNA-damage response, targeted therapy, therapeutic strategy estrogen receptor $\alpha(\mathrm{ER} \alpha)$, progesterone receptor (PR), and human epidermal growth factor receptor-2 (ERBB2/HER2) have been established, and the main breast cancer subtypes are classified according to their molecular profile $(1,2)$. Traditional staging of breast cancer is based on tumor size, lymph node involvement, and presence of metastasis, and recently biologic markers have been incorporated in the 8th edition of the American Joint Committee on Cancer (AJCC), improving the prognostic discrimination over anatomic staging alone (3).

Triple-negative breast cancer (TNBC) is characterized as having $\leq 1 \%$ cellular expression of ER and PR as determined by immunohistochemistry (IHC), and having HER2 expression of 0 to $1+$ by IHC, or $2+$ by IHC and fluorescence in situ hybridization (FISH) negative (i.e. not an amplified gene copy number), according to American Society of Clinical Oncology/College of American Pathologists (ASCO/CAP) guidelines $(4,5)$. TNBCs are comprised of at least four distinct transcriptional subtypes: Two basal subtypes, BL1 and BL2; a mesenchymal subtype $\mathrm{M}$, which is devoid of immune cells; and a luminal androgen receptor (AR) subtype LAR $(1,2)$. TNBC is also subdivided into 6 different subgroups based on molecular heterogeneity: Basal-like; mesenchymal-like; mesenchymal stem-like; luminal AR expression; immunomodulatory; and unstable type (6). TNBC represents approximately $15-20 \%$ of all newly diagnosed breast cancers and is generally a more aggressive disease with a poorer prognosis and higher grade than other types of breast cancer, accounting for $5 \%$ of all cancer-related deaths annually. The median overall survival (OS) for the disease is 10.2 months with current therapies, with a 5-year survival rate of $\sim 65 \%$ for regional tumors and $11 \%$ for those that have spread to distant organs $(7,8)$.

In this review, we discuss current TNBC treatments and key examples of improved clinical benefit, as well as new therapeutic strategies with which to treat the disease.

\section{Current treatment paradigm}

TNBC is chemotherapy sensitive, and this treatment remains the standard of care (SOC). Common chemotherapies include anthracycline (e.g., DNA intercalating agent and topoisomerase II blocker doxorubicin), alkylating agents (e.g., cyclophosamide), an anti-microtubule agent taxane, and an anti-metabolite fluorouracil (5-FU). The current SOC for newly 
diagnosed early TNBC consists of neoadjuvant chemotherapy, followed by surgery. For patients with relapsed/refractory TNBC, there is no standard chemotherapy regimen. Responses to treatment are usually short in duration and followed by rapid relapse, and visceral and brain metastases are common. Available therapies for patients with advanced TNBC include anti-metabolites capecitabine and gemcitabine, non-taxane microtubule inhibitor eribulin, and DNA cross-linker platinums. The median progression-free survival (PFS) with chemotherapy ranges from 1.7 to 3.7 months; the median OS from the onset of metastasis is 10 to 13 months. In clinical trials, patients with advanced TNBC treated with single-agent taxane- or platinum-based chemotherapy had a median PFS of 4 to 6 months and a median OS of 11 to 17 months (9-11).

New treatment options for patients with advanced TNBC have recently emerged, especially in cases where surgery is not an option.

TNBC is more immunogenic than other breast cancer subtypes with tumor-infiltrating lymphocytes (TILs) in its microenvironment. However, TNBC also displays a high level of programmed cell death-ligand 1 (PD-L1) expression $(12,13)$. Thus, immunotherapies targeting the programmed cell death-1 (PD-1) receptor/PD-L1 pathway that maintains immunosuppression in the tumor environment in TNBC have been explored and atezolizumab (anti-PD-L1 antibody) in combination with nanoparticle albumin-bound (nab)-paclitaxel was approved as a first-line therapy by the US Food and Drug Administration (FDA) based on the IMpassion 130 trial (NCT02425891) in 2019. This immuno-chemotherapy became SOC for patients with PD-L1 ${ }^{+}$, unresectable, locally advanced or metastatic TNBC. Note that the survival benefit was exclusively in PD-L1 ${ }^{+}$TNBC patients. The threshold is $1 \%$ PD-L1 expression on infiltrating immune cells by an approved companion diagnostic SP142 IHC assay and 41\% of enrolled patients showed PD-L1-positive expression in the IMpassion130 trial. This is in contrast to studies in other types of cancer which showed benefit for checkpoint inhibitor therapy even in patients with negative PD-L1 expression. In the first interim analysis of IMpassion130, the median PFS was 7.5 vs. 5.0 months with chemotherapy and the median OS was 25.0 vs. 15.5 months with chemotherapy among patients with PD-L1+ tumors (14). In the pre-specified second interim analysis (data cutoff January 2, 2019), the median OS was 25.0 vs. 18.0 months with chemotherapy. Overall, the combination was well-tolerated and immune-related adverse events (AEs) included rash, hypothyroidism, and pneumonitis (15). Another immunotherapy, pembrolizumab (anti-PD-1 antibody), was approved in 2017 as a histology agnostic immunotherapy in all microsatellite instability-high (MSI-H) and/or mismatch repair deficient (dMMR) tumors. This is the first FDA-approved cancer treatment based on a tumor biomarker without regard to the original location of the tumor. However, MSI-H is rare in breast cancer $(<2 \%)(16-18)$.

BRCA1 and BRCA2-deficient tumors exhibit impaired homologous recombination repair (HRR) and synthetic lethality with poly(ADP-ribose) polymerase (PARP) inhibitors $(19,20)$. The FDA approved olaparib and talazoparib in 2018 to treat advanced-stage HER2-negative breast cancer in individuals with a Brcal or Brca2 mutation. The FDA also approved the companion diagnostic test to identify germline Brca-mutated
(gBRCAm) breast cancer patients. Approximately 5\% of patients with breast cancer carry a gBRCAm. Olaparib approval was based on data from the OlympiAD Phase III (NCT02000622) trial comparing olaparib to physician's choice of chemotherapy (capecitabine, vinorelbine or eribulin). Olaparib was associated with a $42 \%$ increase in median PFS as compared to the control group (7 vs. 4 months) in gBRCAm HER2-negative metastatic breast cancer patients with previous chemotherapy (21). There was no statistically significant improvement in OS with olaparib compared to the control group (19.3 vs. 17.1 months), but there was potential OS benefit among patients with no prior chemotherapy for metastatic breast cancer (HR 0.51, 95\% CI 0.29-0.90) (22). Olaparib was generally well-tolerated, with no evidence of cumulative toxicity including the risk of developing anemia during extended exposure. Talazoparib approval was based on data from the EMBRACA Phase III (NCT01945775) trial comparing talazoparib to gemcitabine or to the same physician choice of standard therapy as the OlympiAD trial. Talazoparib increased median PFS by $46 \%$ (8.6 vs. 5.6 months) in gBRCAm HER2-negative locally advanced or metastatic breast cancer patients with previous chemotherapy including an anthracycline and/or taxane. Talazoparib presented with hematologic grade 3-4 AEs (primarily anemia), which occurred in 55 vs. $38 \%$ of the patients with standard therapy, and an improved side-effect profile in patient-reported outcomes (23).

\section{Investigational drugs}

To improve therapeutic benefit in TNBC treatment, various agents have been explored in clinical studies. They include immuno- and targeted-therapies in the networks of tumor-stroma, DNA damage response (DDR), cell surface or intracellular receptors, and signaling pathways as well as cell surface markers for selective drug delivery, and antibody-drug conjugates (ADCs) (Fig. 1). As of March 2020, 399 ongoing studies for TNBC have been listed on ClinicalTrials.gov and select Phase III studies are listed in Table I.

Immunotherapy: Immune checkpoint. TILs are frequent in TNBC, correlate with increased pathologic complete response (pCR) to neoadjuvant chemotherapy, and are predictive of disease-free survival (DFS) and OS in early-stage TNBC (24-26). Expression of immune regulatory checkpoints is an adaptive method of tumor resistance to infiltrating lymphocytes within the tumor microenvironment. Multiple strategies have been used to enhance the response to PD-1/PD-L1 blockade in pre-clinical and early clinical studies, including several intratumoral immune modulators and targeted agents (27). The activity of immunotherapy, such as immune checkpoint inhibitors, can be enhanced by chemotherapeutic agents through the stimulation/release of antigens, thus leading to promotion of immunogenic cell death. Currently, clinical trials investigating the use of immune checkpoint inhibitors are ongoing either as a single agent or in various combinations with other agents beyond the metastatic setting and even in the first-line setting (28).

Neoadjuvant treatment. Studies determining benefit from neoadjuvant checkpoint inhibitor therapy have yielded mixed outcomes. Neoadjuvant chemotherapy with pembrolizumab 


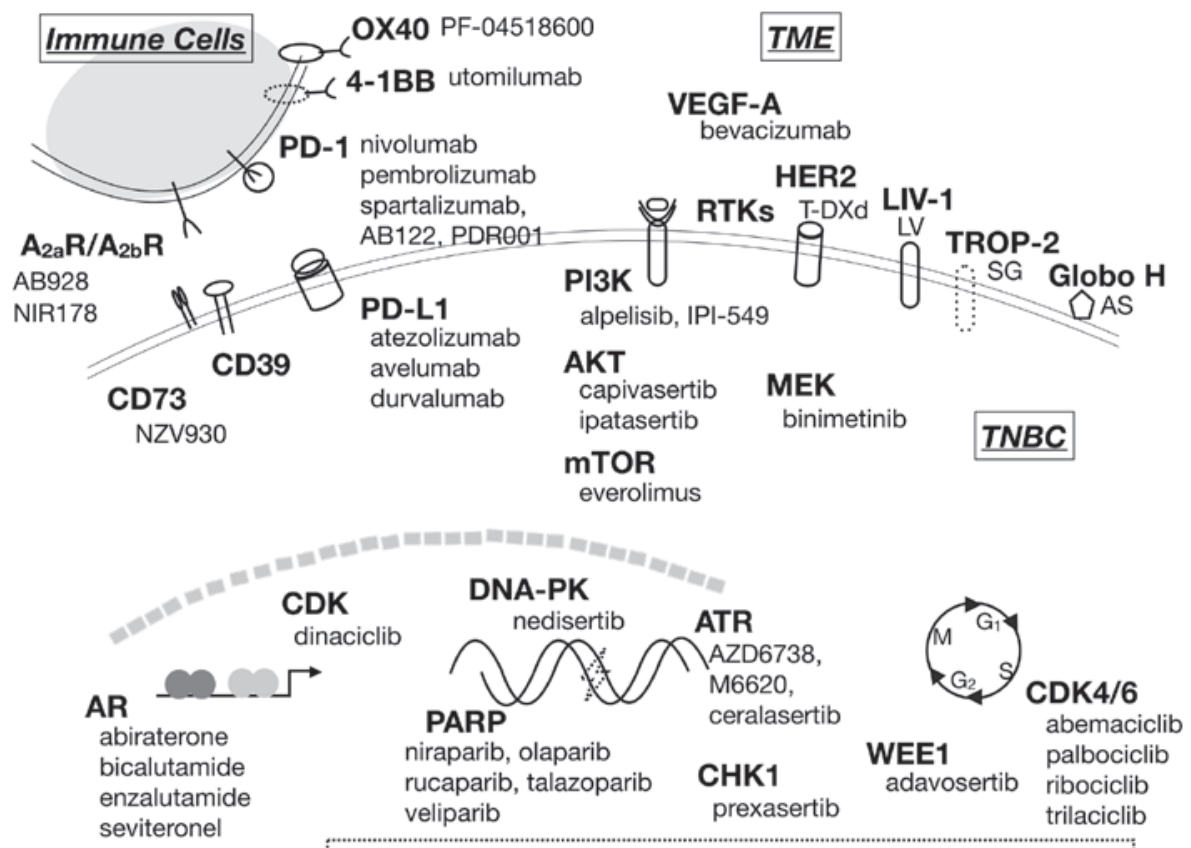

doxorubicin, cyclophosamide, carboplatin, taxane, eribulin, paclitaxel, nab-paclitaxel, vinorelbine, fluorouracil, capecitabine, gemcitabine

Figure 1. Immuno- and targeted-therapies in key TNBC clinical studies. Various agents in the networks of TNBCs and immune cells have been explored, as well as tumor-stroma interactions in the tumor microenvironment (TME). Targets and agents relevant to immune checkpoint, cell surface or intracellular receptors, signaling pathways, DNA damage response, and cell cycle checkpoint are shown. Various chemotherapy agents are listed in the box. AS, Adagloxad simolenin); LV, Ladiratuzumab vedotin; SG, Sacituzumab govitecan-hziy; T-DXd, tastuzumab deruxtecan; TNBC, triple-negative breast cancer; $\mathrm{A}_{2 \mathrm{a}} \mathrm{R}$, adenosine 2A receptor; $\mathrm{A}_{2 \mathrm{~b}} \mathrm{R}, 2 \mathrm{~B}$ receptor; PD-1, programmed cell death-1; PD-L1, programmed cell death-ligand 1; VEGF-A, vascular endothelial growth factor A; RTKs, receptor tyrosine kinases; PARP, poly(ADP-ribose) polymerase; CDK, cyclin-dependent kinase; CD, cluster of differentiation; ATR ataxia telangiectasia and Rad3-related kinase; CHK1, checkpoint kinase 1; DNA-PK, DNA-dependent protein kinase; AR, androgen receptor; PI3K, phosphatidylinositol 3-kinase.

have demonstrated manageable safety and promising antitumor activity for patients with early-stage TNBC in the KEYNOTE-173 Phase 1b (NCT02622074) (29) and I-SPY2 Phase II (NCT01042379) trials (30). The KEYNOTE-522 Phase III trial (NCT03036488) further explored neoadjuvant chemotherapy with or without pembrolizumab followed by surgery and pembrolizumab or placebo adjuvantly. The neoadjuvant combination showed a significantly higher pCR rate than the placebo-chemotherapy group (65 vs. 51\%). Note that a similar pCR benefit ( $\sim 15 \%)$ in both the PD-L-positive and -negative subgroups was observed, suggesting that neoadjuvant pembrolizumab may benefit patients regardless of PD-L1 levels. This is different from the advanced setting where only the PD-L1-positive patients benefit from atezolizumab. The toxicity profiles were as expected for each treatment, with similar rates (78 vs. $73 \%$ ) of grade $\geq 3$ treatment-related AEs (TRAEs) (31).

NeoTRIPaPDL1 Phase III (NCT02620280) trial also explored neoadjuvant chemotherapy with or without atezolizumab followed by surgery and four cycles of an anthracycline regimen. However, in this trial for patients with early-stage high-risk or locally advanced unilateral breast cancer there was no improvement in $\mathrm{pCR}$ with the combination therapy (44 vs. $41 \%$ with the control arm) (32). Note that the neoadjuvant chemo-regimen was different from KEYNOTE-522 which included another round of chemotherapy following carboplatin and nab-paclitaxel. The difference in the targets, PD-1 for pembrolizumab vs. PD-L1 for atezolizumab, may also have contributed to the different outcomes. Another Phase III (NCT03197935) trial, IMpassion031 study also explored atezolizumab in combination with chemotherapy (nab-paclitaxel followed by doxorubicin and cyclophosphamide) in comparison to placebo plus chemotherapy in the neoadjuvant setting. Treatment with atezolizumab continued adjuvantly for those in the combination arm of the study (33). The primary endpoint was $\mathrm{pCR}$.

In the advanced setting. As a first-line treatment option for patients with locally recurrent, inoperable or metastatic TNBC, pembrolizumab was evaluated in combination with investigator's choice of chemotherapy (i.e. nab-paclitaxel, paclitaxel or gemcitabine/carboplatin), compared to placebo plus chemotherapy (KEYNOTE-355 Phase III trial, NCT02819518). A significant PFS benefit with the pembrolizumab-chemo combination in patients whose tumors expressed PD-L1 (CPS $\geq 10)$ was reported (9.7 vs. 5.6 months for chemotherapy alone) (34). The study is currently in progress to evaluate OS, the other primary endpoint of the trial.

In contrast to other studies of immunotherapy combined with SOC chemotherapy, the Tonic trial (NCT02499367) in metastatic TNBC was based on an adaptive trial design that explores a sequential treatment with anti-PD-1 antibody nivolumab after 2 weeks of chemotherapy or radiotherapy. The hypothesis is that short-term treatment induces a more favorable tumor microenvironment that would enhance sensitivity to immune checkpoint blockade in TNBC. The highest overall 


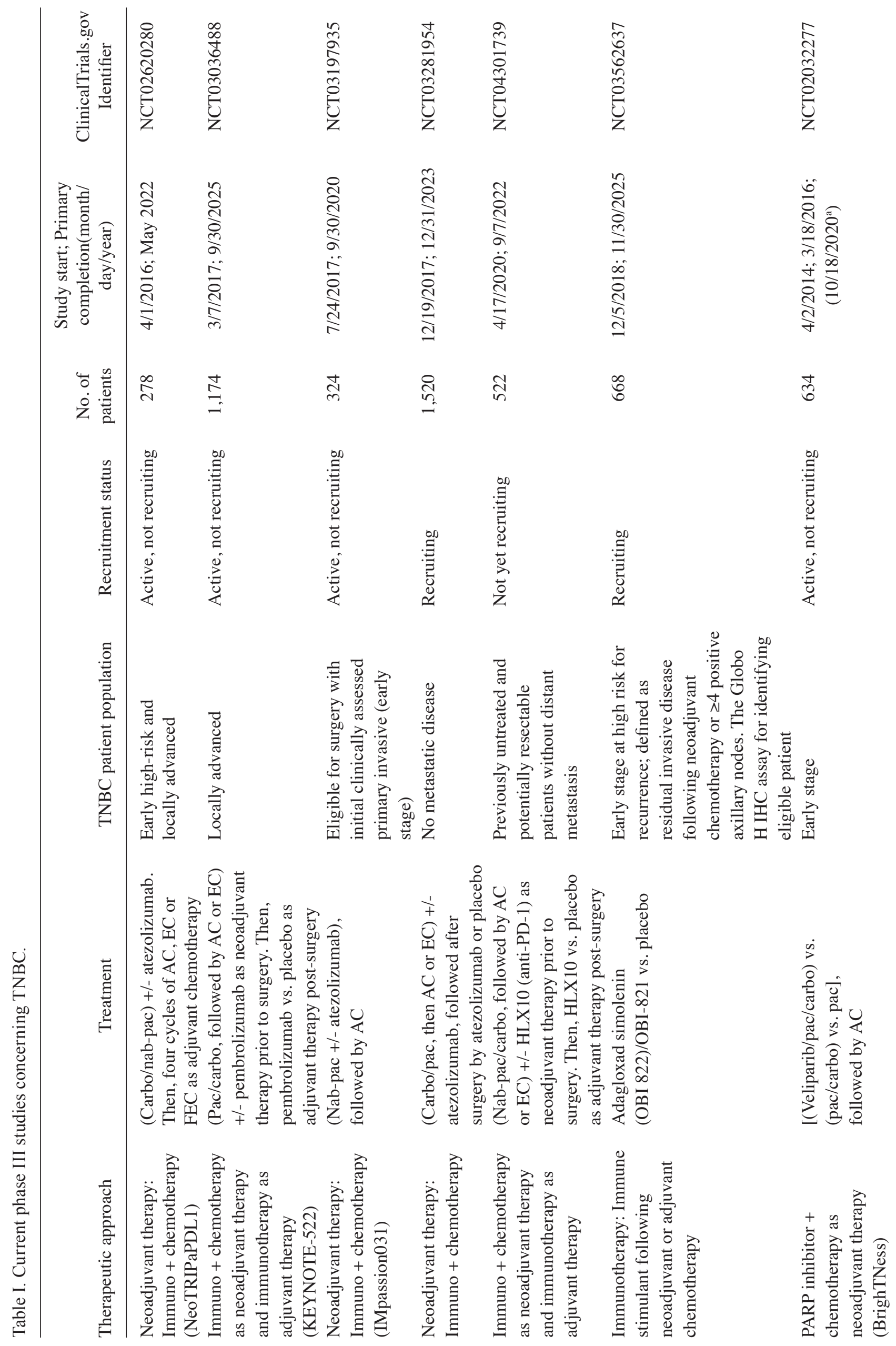




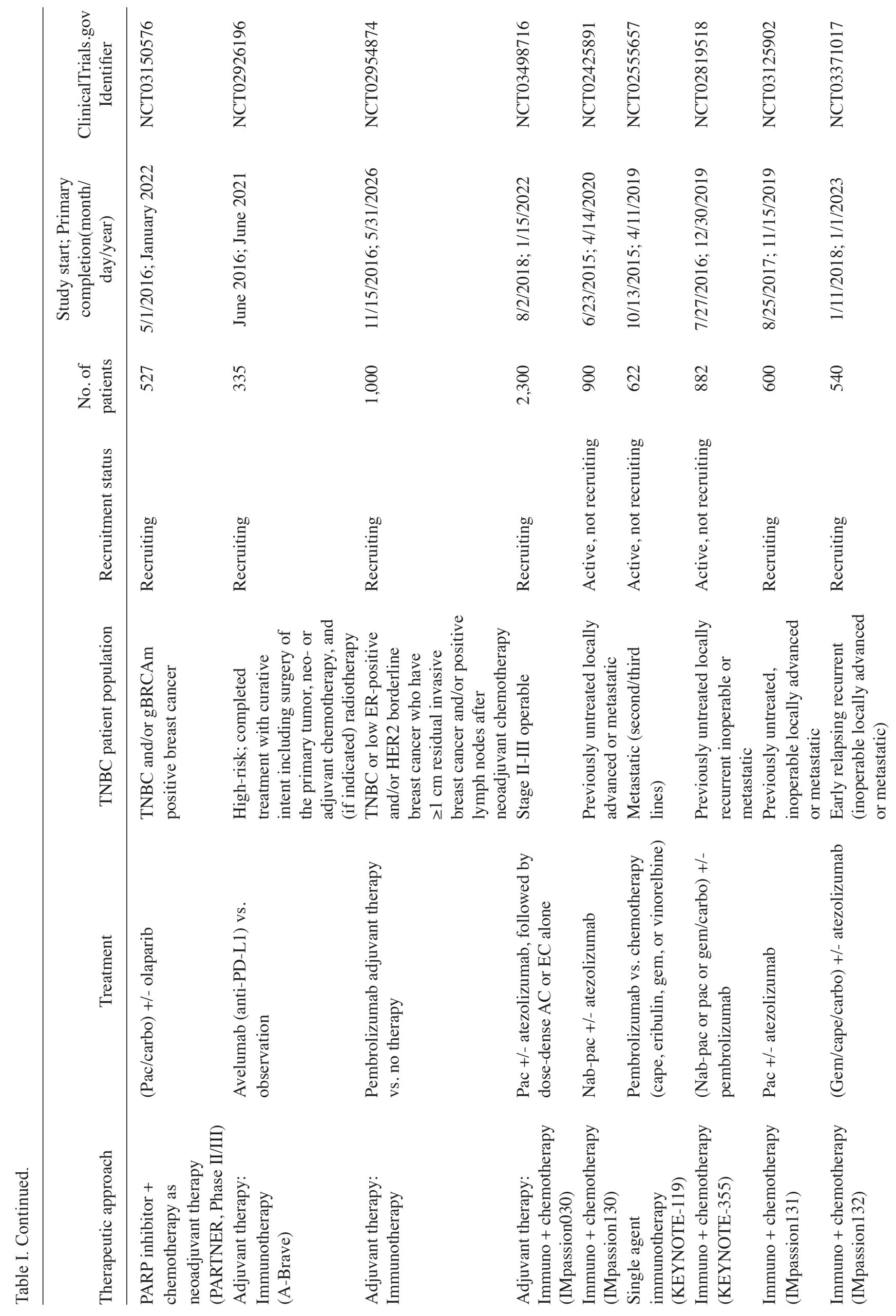




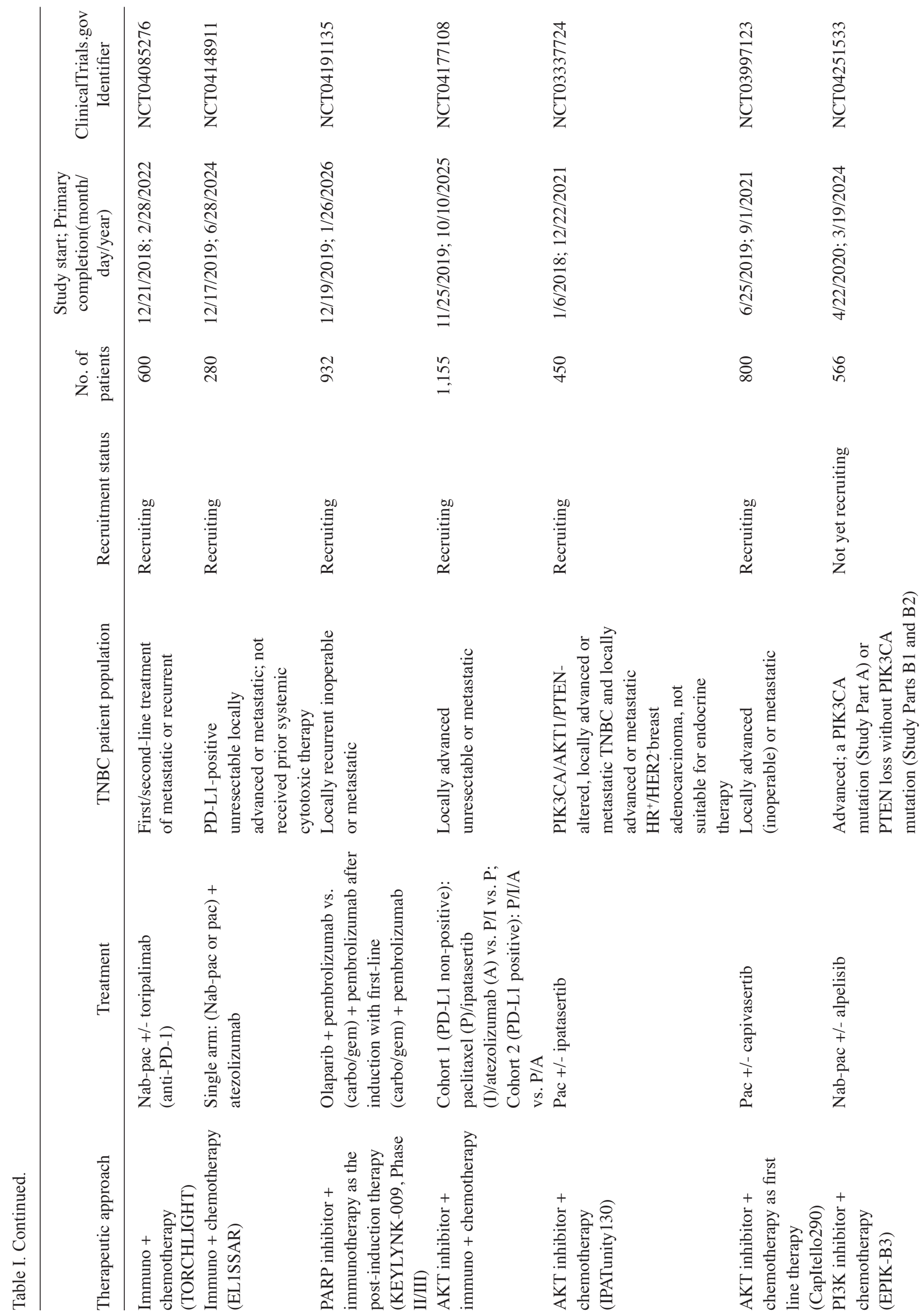




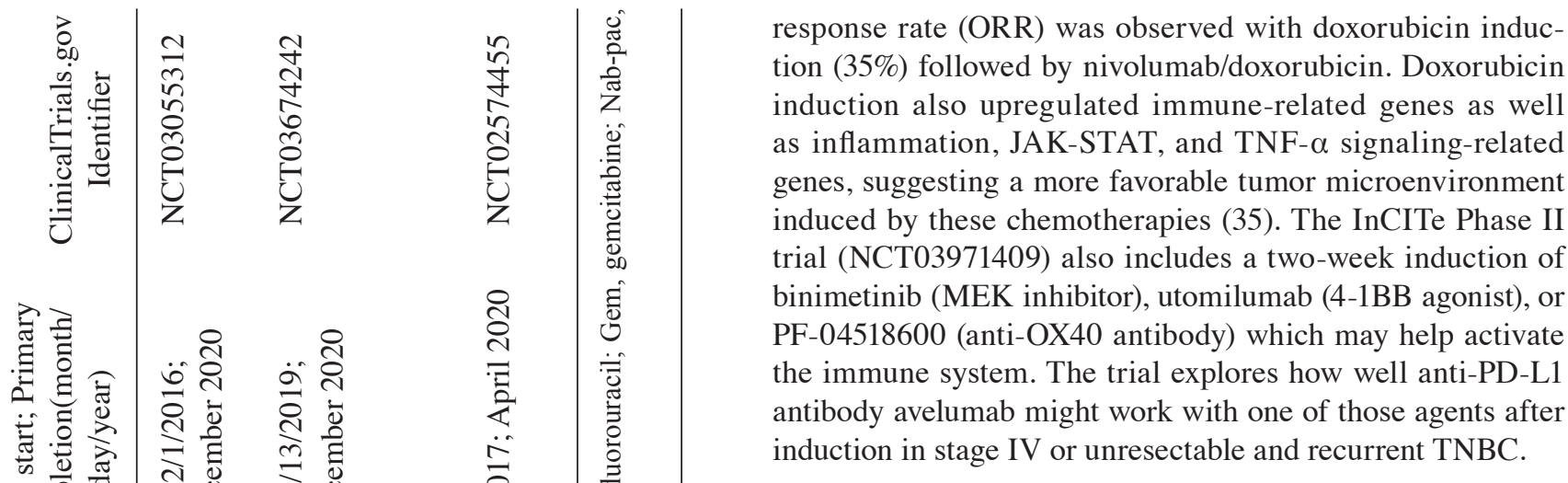

Immunotherapy: Adenosine pathway. Adenosine is catabolized from ATP and often overproduced and released by tumor cells. It is also converted from extracellular nucleotides by the plasma membrane protein, cluster of differentiation 73 (CD73), which is upregulated in many cancer types $(36,37)$. The excess adenosine in the tumor microenvironment activates the adenosine $2 \mathrm{~A}$ receptor $\left(\mathrm{A}_{2 \mathrm{a}} \mathrm{R}\right)$ and $2 \mathrm{~B}$ receptor $\left(A_{2 b} R\right)(38,39)$ which are highly expressed on the cell surfaces of lymphocytes and myeloid cells, respectively, leading to immunosuppressive effects (Fig. 2). Targeting these receptors and enzymes could lead to reactivation of antitumor immunity by abrogating the inhibitory effect on the immune system and enhancing the cytotoxic $\mathrm{T}$ lymphocyte (CTL)-mediated immune response $(40,41)$.

Combinations of adenosine pathway inhibitors and immune checkpoint inhibitors have been explored in clinical trials. NZV930 (SRF373) is an anti-CD73 monoclonal antibody that binds to CD73 on tumor cells, leading to internalization of CD73, thereby preventing CD73-mediated conversion of extracellular AMP to adenosine. A Phase $\mathrm{I} / \mathrm{Ib}$ study (NCT03549000) is underway to evaluate NZV930 alone and in combination with PD-1 inhibitor PDR001 and/or $\mathrm{A}_{2 \mathrm{a}} \mathrm{R}$ antagonist NIR178 in patients with advanced malignancies including TNBC. NIR178 is an antagonist of $A_{2 \mathrm{a}} \mathrm{R}$, blocking adenosine $/ \mathrm{A}_{2 \mathrm{a}} \mathrm{R}$-mediated inhibition of $\mathrm{T}$ lymphocytes. A Phase II study (NCT03207867) is underway for NIR178 in combination with PD-1 inhibitor spartalizumab in multiple solid tumors and diffuse large B-cell lymphoma (DLBCL) to assess if the addition of the adenosine antagonist improves the efficacy of PD-1 inhibition. A dual adenosine $A_{2 a} R / A_{2 b} R$ receptor antagonist, $\mathrm{AB} 928$, is currently being evaluated in a Phase I study (NCT03629756) in combination with the PD-1 inhibitor AB122 in patients with advanced malignancies. Early results show a favorable safety profile of AB928 combination therapy and predictable PK/PD correlation (42).

DNA-damage response: PARP. Approximately $60-70 \%$ of breast cancer patients with an inherited Brcal/2 mutation are TNBC subtype and $10-30 \%$ of TNBC patients harbor a Brca pathogenic variant $(43,44)$. A condition defined as 'BRCAness' (45), which includes mutations in HRR genes through genetic or epigenetic inactivation, leads to susceptibility to both platinum and PARP inhibitors. Various PARP inhibitors (e.g. veliparib, niraparib, and rucaparib as well as olaparib and talazoparib) have been assessed in the neoadjuvant and adjuvant settings and in combination with other agents. 


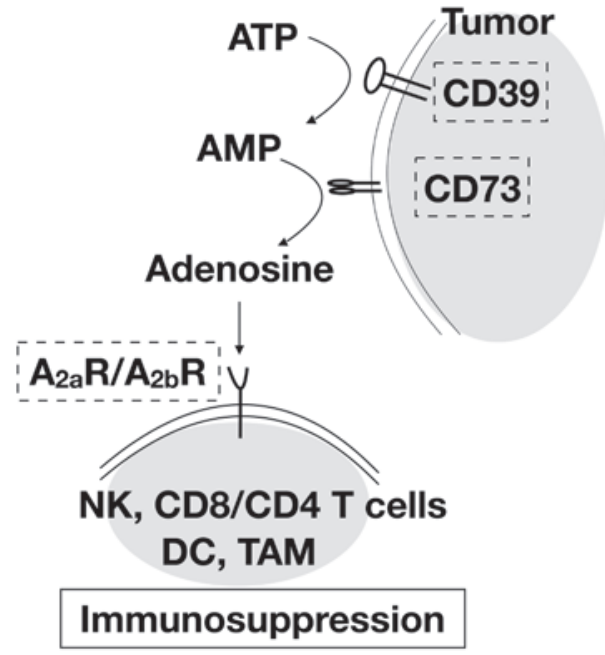

Figure 2. ATP-adenosine pathway. Adenosine is generated from ATP by CD39 and CD73. It binds to A2 receptors on immune cells and blocks T cell priming, expansion, and activation, natural killer (NK) cell degranulation, dendritic cell (DC) maturation and activation, and tumor-associated macrophage (TAM) M1 polarization, thus leading to immunosuppression. ATP, adenosine triphosphate; AMP, adenosine monophosphate; CD, cluster of differentiation.

Neoadjuvant and adjuvant settings. A PARP inhibitor appears to have efficacy for neoadjuvant treatment of patients with gBRCAm TNBC. Talazoparib achieved encouraging pCR in patients with gBRCAm breast cancer, including TNBC, and $\mathrm{HR}^{+}$breast cancer, as a neoadjuvant single-agent without the addition of chemotherapy (46). Currently a larger, multicenter, neoadjuvant Phase II trial (NCT03499353) is ongoing. However, the addition of a PARP inhibitor to standard neoadjuvant chemotherapy was found to be not beneficial. In the BrighTNess Phase III trial (NCT02032277) the addition of PARP inhibitor veliparib to carboplatin and paclitaxel followed by doxorubicin and cyclophosphamide did not improve pCR whereas the addition of veliparib and carboplatin to paclitaxel did. Therefore, the addition of carboplatin but not veliparib to paclitaxel was proposed as a potential component of neoadjuvant chemotherapy for patients with high-risk TNBC (47).

PARP inhibitors have also been studied as an adjuvant single-agent therapy. The OlympiA Phase III trial (NCT02032823) was designed to assess olaparib in patients with gBRCAm and high-risk HER2-negative breast cancer who completed definitive local treatment and neoadjuvant or adjuvant chemotherapy. The primary outcome measure will be invasive DFS with a time frame of up to 10 years.

In combination with immunotherapy. A crosstalk exists between PARP inhibition and the PD-L1/PD-1 immune checkpoint axis. PARP inhibitors upregulate PD-L1 expression on tumor cells by inhibiting glycogen synthase kinase 3 beta (GSK3 $\beta$ ) and activating the cGAS-STING pathway (48). Thus, primary/acquired resistance to PARP inhibitors seems to be associated with the development of immune evasion mechanisms. Multiple clinical studies are underway to assess synergy between therapeutic strategies of PARP inhibition and immune checkpoint blockers.

In platinum-resistant, advanced, or metastatic TNBC, niraparib combined with pembrolizumab
(TOPACIO/KEYNOTE-162 Phase II trial, NCT02657889) showed higher response rates in patients with tumor Brca mutations (tBRCAm): ORR of $28 \%$ in all (biomarker-unselected) patients vs. $60 \%$ for tBRCAm patients. The combination therapy was safe with a tolerable safety profile (49).

In MEDIOLA Phase I/II trial (NCT02734004) the combination of olaparib and durvalumab showed ORR of $63 \%$ in a cohort of patients with gBRCAm metastatic breast cancer (50). In the I-SPY 2 Phase II study (NCT01042379), adding the same combination to neoadjuvant paclitaxel led to improved pCR rates in patients with high-risk, HER2-negative stage II/III breast cancer compared with single-agent paclitaxel. In those with TNBC, the pCR rate was 47 vs. $27 \%$ with paclitaxel alone. AEs were consistent with the known safety profiles of each agent alone (51). In metastatic TNBC, the efficacy of induction treatment of olaparib followed by the combination treatment of olaparib and durvalumab is being assessed in a Phase II study (NCT03801369) (52). Patients with $\leq 2$ prior chemotherapy regimens for metastatic breast cancer are eligible, but patients with gBRCAm TNBC are excluded. The primary end point is ORR.

The DORA Phase II trial (NCT03167619) is evaluating olaparib as a maintenance therapy with or without durvalumab in patients with advanced TNBC who achieve at least stable disease after 3 cycles of platinum-based chemotherapy. Another study of a PARP inhibitor as a maintenance therapy, KEYLYNK-009 Phase II/III trial (NCT04191135), is underway in metastatic TNBC to assess the efficacy of olaparib plus pembrolizumab vs. chemotherapy plus pembrolizumab after induction with first-line chemotherapy plus pembrolizumab (53).

In combination with DDR-HRR pathway inhibitors. Resistance to PARP inhibitors can occur in certain cancer contexts by various mechanisms, including increased HRR capacity and decreased cell cycle progression and DNA replication stress. RAD51 overexpression has been observed in a wide range of human cancers, particularly TNBCs and serous ovarian cancers $(54,55)$. Upregulation of RAD51 in BRCA1-defective cells is also associated with resistance to PARP inhibitor $(56,57)$. Inhibitors of key mediators of DNA repair and replication, such as ataxia telangiectasia mutated kinase (ATM), ataxia telangiectasia and Rad3-related kinase (ATR), checkpoint kinase 1 (CHK1) and checkpoint kinase 2 (CHK2), DNA-dependent protein kinase (DNA-PK), and WEE1 kinase (Fig. 3) have been assessed to determine if they can sensitize tumor cells to treatment with PARP inhibitors, as these inhibitors were found to prevent the accumulation of RAD51 in TNBC (58).

The VIOLETTE Phase II study (NCT03330847) was set up to assess the combinatory inhibition of PARP and a component of the ATR-CHK1-WEE1 axis. Olaparib with DDR kinase ATR inhibitor AZD6738 was compared to olaparib monotherapy in the second- or third-line setting of metastatic TNBC. Patients were stratified by Brca and HRR gene mutation status and the primary endpoint was PFS (59). The study also included a combination arm of olaparib with the first-in-class WEE1 inhibitor adavosertib. WEE1 inhibitor was found to potentiate the activity of DNA-damaging agents in preclinical TNBC models $(60,61)$ and its potential clinical 


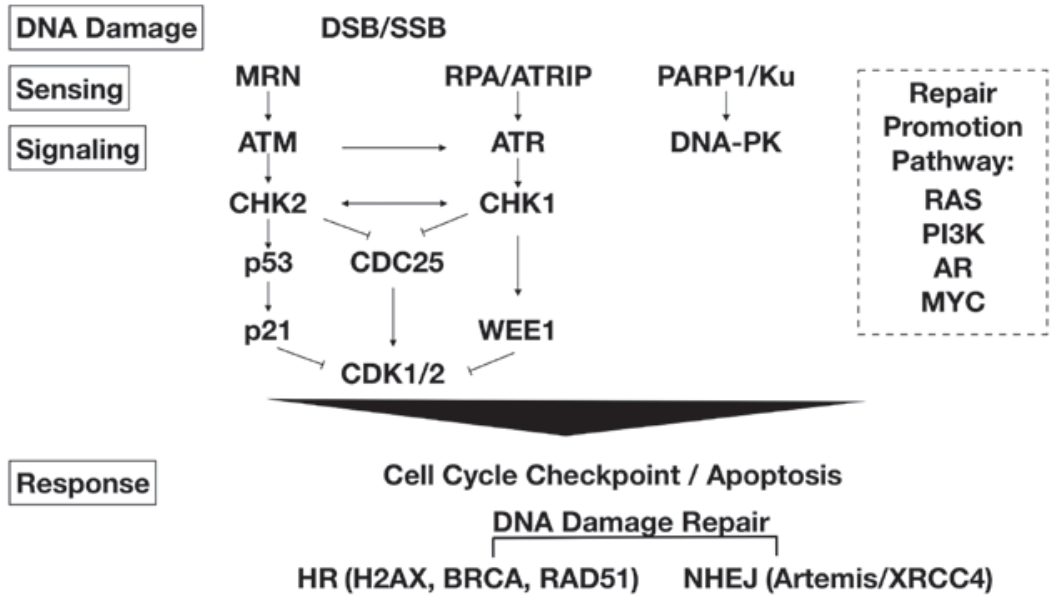

Figure 3. DNA damage response pathways. Double-strand breaks (DSB) or single-strand breaks (SSB) activate DNA damage response (DDR) pathways, leading to cell cycle arrest and DNA repair or cell death depending on cell context. PARP1 senses DNA breaks and is involved in SSB repair. Oncogenic pathways including RAS, PI3K, AR, and MYC signaling can affect HR repair activity and contribute to resistance to PARP inhibitor treatment. MRN, MRE11-RAD50-NBS1 complex; ATRIP, ATP interacting protein; HR, homologous recombination; NHEJ, nonhomologous end joining; H2AX, histone H2AX; XRCC4, X-ray repair cross-complementing protein 4; ATR, ataxia telangiectasia and Rad3-related protein; CHK1/2, checkpoint kinase 1/2; CDK1/2, cyclin-dependent kinase 1/2; DNA-PK, DNA-dependent protein kinase; AR, androgen receptor; PI3K, phosphatidylinositol 3-kinase.

value was observed in a Phase I study in patients with Brca mutations (62). However, the combination treatment arm of olaparib and adavosertib was discontinued in the VIOLETTE study and patients were offered the opportunity to continue treatment on olaparib monotherapy. The CHK1 inhibitor prexasertib in combination with olaparib was also explored in early clinical trials (63), but development of prexasertib was discontinued by the sponsor in 2019 .

\section{Intracellular signaling pathway targets}

$P I 3 K / A K T$ pathway. A wide range of malignancies including TNBC show dysregulated phosphatase and tensin homolog (PTEN)/phosphoinositide 3-kinases (PI3K)/protein kinase B $(\mathrm{AKT}) / \mathrm{mammalian}$ target of rapamycin (mTOR) signaling due to mutations in multiple signaling components. Loss of PTEN, a negative regulator of AKT, was found to be correlated with decreased T-cell infiltration at tumor sites in patients, and inhibition of the PI3K-AKT pathway re-sensitized to T-cell-mediated immunotherapy (64). As the PI3K/AKT pathway has emerged as a potential mechanism of resistance to immunotherapy and chemotherapy, multiple clinical trials have assessed inhibitors of the various pathway components.

Alpelisib is an oral PI3K inhibitor that selectively inhibits p1 $10 \alpha$. It showed efficacy in targeting Pik3ca-mutated breast cancer (65) and was FDA approved in 2019 in combination with fulvestrant for postmenopausal women and men, with $\mathrm{HR}^{+}$, HER2-negative, Pik3ca-mutated, advanced or metastatic breast cancer following progression on or after an endocrine-based regimen. For patients with advanced TNBC, the EPIK-B3 Phase III trial (NCT04251533) is planned with study start date of April 2020 to assess alpelisib in combination with nab-paclitaxel. Patients have Pik3ca mutations or PTEN loss with $\leq 1$ prior line of therapy for metastatic disease.

IPI-549 is a selective PI3K-gamma inhibitor targeting immune-suppressive tumor-associated myeloid cells. The MARIO-3 Phase II study (NCT03961698) was designed to explore the addition of IPI-549 to the FDA approved regimen atezolizumab/nab-paclitaxel in front-line TNBC. Cohort A will be composed of patients with locally advanced, metastatic TNBC, which will include two sub-cohorts based on PD-L1 IHC status. The primary objective is CR rate.

Ipatasertib and capivasertib are pan-AKT inhibitors that bind to all three isoforms of AKT. Both are now in Phase III trials evaluating the efficacy of combination with paclitaxel as first-line therapy for locally advanced or metastatic TNBC. In the LOTUS Phase II trial, adding ipatasertib to first-line paclitaxel improved PFS, particularly in patients with PTEN/PI3K/AKT-altered tumors (HR, 0.44) (66). In this subgroup of patients, median OS was 23.1 vs. 16.2 months with placebo (HR, 0.65) (67). To confirm the findings from LOTUS, the IPATunity130 Phase III trial (NCT03337724) is evaluating ipatasertib + paclitaxel for PTEN/PI3K/AKT-altered advanced TNBC or $\mathrm{HR}^{+}$, HER2-negative breast cancers. The primary endpoint is PFS (68). An independent trial also supported the potential benefit for addition of AKT inhibitor to chemotherapy. In the PAKT Phase II study (NCT02423603), addition of the oral AKT inhibitor capivasertib to first-line paclitaxel resulted in significantly longer PFS and OS in patients with advanced TNBC, especially in patients with PTEN/PI3K/AKT-altered tumors. The median PFS duration was 5.9 vs. 4.2 months with placebo, meeting the predefined significance level, and better benefit in patients with PTEN/PI3K/AKT-altered tumors with median PFS of 9.3 months (HR, 0.30). The median OS was prolonged by 6.5 months with capivasertib (69). The most common AEs of grade $\geq 3$ were diarrhea, infection, rash, and fatigue, similar to those observed with ipatasertib in the LOTUS trial. The CAPItello-290 Phase III trial (NCT03997123) is underway and the primary endpoints are PFS and OS (70).

Efficacy of immunotherapy was also found to be enhanced by AKT inhibitors as a first-line therapy for locally advanced/metastatic TNBC. Phase Ib study (NCT03800836) was designed to evaluate the triplet combination of ipatasertib (I), atezolizumab (A), and paclitaxel or nab-paclitaxel (P). Preliminary efficacy and safety data up to January 5, 2019 showed that the triplet regimen had promising antitumor 
activity (73\% confirmed ORR), irrespective of biomarker PD-L1 status or PTEN/PI3K/AKT alteration status, and manageable toxicity (71). In Phase III trial (NCT04177108), patients were enrolled in two cohorts according to PD-L1 status: Cohort 1 for PD-L1-negative tumors and cohort 2 for PD-L1-positive tumors. Three arms, $\mathrm{P}+\mathrm{I}+\mathrm{A}$ vs. P + I vs. P, will be evaluated in cohort 1 and 2 arms, $\mathrm{P}+\mathrm{I}+\mathrm{A}$ vs. $\mathrm{P}+\mathrm{A}$, will be evaluated in cohort 2 .

$C D K 4 / 6 / R b / E 2 F$ pathway. The $\mathrm{G}_{1}-\mathrm{S}$ phase checkpoint of the cell cycle is regulated by CDK4/6 activity which is controlled by their binding partners D-type cyclins and p16 INK4 inhibitor. The active CDK4/6-cyclin D complex phosphorylates the retinoblastoma $(\mathrm{Rb})$ protein, thereby activating $\mathrm{E} 2 \mathrm{~F}$ function and transition from $G_{1}$ to $S$ phase of the cell cycle (72). The FDA approved CDK4/6 inhibitors palbociclib, ribociclib, and abemaciclib based on improvements in PFS for the treatment of $\mathrm{ER}^{+}, \mathrm{HER} 2$-negative advanced or metastatic breast cancer in combination with an endocrine therapy. TNBCs with a $\mathrm{Rb}^{+}$, p16 INK4-negative profile might represent the subpopulation of TNBC suitable for treatment with CDK4/6 inhibitors.

Preclinical combination studies of CDK4/6 inhibitors with chemotherapy suggest that the timing and sequence of drug exposure/drug delivery schedule might play a critical role in drug activity, and the evaluation of different schedules of treatment may represent a new approach $(73,74)$. The hypothesis was that reversible $\mathrm{G}_{1}$ arrest of palbociclib could synchronize tumor cells in the cell cycle and following their re-entry later would ensure a higher fraction in mitosis $(\mathrm{M})$ phase when exposed to paclitaxel. In the first combination trial for palbociclib and paclitaxel (NCT01320592) an alternative dosing schedule was feasible and safe, without evidence of additive toxicity in $\mathrm{Rb}^{+}$ breast cancer regardless of subtype (75). Phase I follow-up trial (NCT02599363) of ribocilcib and weekly paclitaxel is in progress in patients with $\mathrm{Rb}^{+}$advanced breast cancer. In this study, pharmacodynamic, histologic, and imaging biomarkers will be utilized to confirm synchronization and schedule and identify a patient population that benefits from this treatment approach.

The standard chemotherapy regimen causes treatment-limiting cumulative myelosuppression that may compromise antitumor efficacy in TNBC. CDK4/6 inhibitors induce transient $G_{1}$ arrest in immune cells and hematopoietic stem and progenitor cells, potentially helping to preserve T-cell function and bone marrow. To test this hypothesis, an investigational CDK4/6 inhibitor trilaciclib in combination with gemcitabine and carboplatin was explored to evaluate benefit for patients with $\leq 2$ prior chemotherapy regimens in metastatic TNBC. Phase II trial (NCT02978716) was negative for a safety-related primary endpoint (i.e. no difference in the frequency or duration of severe grade 4 neutropenia). However, the median OS was improved by more than $60 \%$, which was likely due to increased chemotherapy duration and exposure. Trilaciclib-treated patients also had a higher number of activated $\mathrm{CD}^{+} \mathrm{T}$ cells over the first 5 cycles of chemotherapy, which potentially enhanced antitumor immunity (76).

MYC and CDK. Transcription factor c-MYC triggers selective gene expression to promote cell growth and proliferation. It is amplified in several different cancer types including TNBC, functioning as a proto-oncogene (77). c-MYC compensates for BRCA loss by upregulating HRR through increased RAD51 expression $(55,78)$. TNBC patients with high c-MYC and RAD51 expression exhibit poor prognosis and less favorable response to chemotherapy and PARP inhibitors $(55,57,79)$. c-MYC blockade in TNBC was found to be synthetic lethal with PARP inhibitors, independent of BRCA status (80). c-MYC pathway activation in TNBC is also synthetic lethal with $C D K$ inhibition (81). Dinaciclib is a pan-CDK (CDK1/2/5/9) inhibitor and the combination with PARP1 inhibitor veliparib is currently being pursued in patients with advanced solid tumors for which no curative therapy exists (Phase I trial, NCT01434316). Dinaciclib induced immunogenic cell death (ICD) but also increased expression of PD1 on tumor-infiltrating $\mathrm{T}$ cells and expression of PD-L1 on tumor cells, thus limiting its antitumor effect in preclinical studies. However, dinaciclib inhibits tumor growth in combination with anti-PD-1 (82). Phase Ib trial (NCT01676753) was designed to evaluate the efficacy of combined dinaciclib and pembrolizumab in patients with metastatic or locally advanced and unresectable TNBC. Its clinical benefit rate was $47 \%$ in preliminary efficacy analysis and high c-MYC expression correlated significantly with clinical response, warranting further validation of c-MYC as a predicative biomarker of response to $\mathrm{CDK} /$ checkpoint inhibitors (83).

AR antagonists. The androgen receptor (AR) is an intracellular steroid receptor that dimerizes and translocates to the nucleus after binding androgen ligands. In the nucleus, AR binds to androgen response elements to promote target gene transcription in a tissue-specific manner. AR can also be activated in a ligand-independent manner through crosstalk with key signaling pathways, including PI3K/AKT and ERK (84). AR is involved in cell cycle regulation and the epithelial-to-mesenchymal transition (EMT) $(85,86)$. AR has emerged as a new biomarker and a potential therapeutic target in TNBC. AR is expressed in $\geq 40 \%$ of TNBCs and its expression level varies considerably among TNBC molecular subtypes. It has been associated with favorable prognosis, with better DFS and higher OS in the LAR subtype $(87,88)$. However, patients with $\mathrm{AR}^{+} \mathrm{TNBC}$ have a decreased chance of achieving $\mathrm{pCR}$ to neoadjuvant chemotherapy and the LAR subtype has been linked to poorer response to chemotherapy compared to other TNBC patients (89-91). Multiple selective AR inhibitors have been approved by the FDA for the treatment of prostate cancer and are currently part of standard care (92). The role of the AR in signaling pathways in TNBC is still not clear and clinical studies are underway to provide more insight into the role of the AR as well as to assess whether AR targeting is a valuable therapeutic strategy in TNBC.

The first proof-of-concept trial of AR-targeted treatment established activity of the first-generation AR antagonist bicalutamide in patients with advanced $\mathrm{AR}^{+} \mathrm{TNBC}$. The TBCRC 011 Phase II trial (NCT00468715) showed a modest clinical benefit rate (CBR) of $19 \%$ at 6 months and a median PFS duration of 12 weeks (93).

$\mathrm{AR}^{+} \mathrm{TNBC}$ expresses a luminal profile with intact $\mathrm{Rb}$ protein, the target of CDK4/6 activity. Thus, CDK4/6 inhibitors may increase the efficacy of AR antagonists in metastatic $\mathrm{AR}^{+} \mathrm{TNBC}$. The single group Phase I/II trial (NCT02605486) 
was carried out to explore this hypothesis. The combination of palbociclib and bicalutamide was well-tolerated with no unexpected toxicity (94). It also met its prespecified efficacy endpoint as measured by PFS with 11 patients (31 evaluable patients) at 6 months (95).

As one of the second-generation anti-androgen therapies, abiraterone is a steroidal CYP17 inhibitor with potent hydroxylase activity, targeting androgen biosynthesis. The French Breast Cancer Intergroup (UCBG) 12-1 Phase II trial (NCT01842321) was designed to evaluate abiraterone acetate (AA) with its requisite concomitant medication prednisone in $\mathrm{AR}^{+}$advanced or metastatic TNBC. Androgen deprivation by AA resulted in $20 \%$ of the 6 -month CBR. This treatment appeared to be beneficial for some patients with molecular apocrine tumors, a subtype that expresses AR but not $\mathrm{ER} \alpha(96)$. Considering that prednisone stimulates the glucocorticoid receptor (GR), which is expressed in approximately $25 \%$ of TNBCs, GR activity might limit the efficacy of AA.

Seviteronel is an investigational lyase-selective non-steroidal CYP17 inhibitor that targets androgen and estrogen production. The CLARITY-01 Phase I/II trial (NCT02580448) was set up to evaluate seviteronel in locally advanced or metastatic TNBC or $\mathrm{ER}^{+}$breast cancer. It revealed that seviteronel was generally well-tolerated and provided clinical benefit. A total of 26 and $11 \%$ of patients reached at least a CBR at 4 and 6 months, respectively. Levels of circulating tumor cells (CTCs) also decreased $(97,98)$.

A second-generation AR antagonist enzalutamide not only competitively binds to the AR ligand-binding domain, but also inhibits nuclear translocation of AR, DNA binding, and coactivator recruitment. Phase II single arm study (NCT01889238) assessed the efficacy of enzalutamide in patients with locally advanced or metastatic, $\mathrm{AR}^{+} \mathrm{TNBC}$. The primary endpoint was CBR at 16 weeks, which was $25 \%$ in the intention-to-treat (ITT) population and $33 \%$ in the evaluable subgroup whose tumors expressed $\geq 10 \%$ nuclear AR. The only treatment-related grade 3 or greater $\mathrm{AE}$ occurring in $\geq 2 \%$ of patients was fatigue (3.4\%) (99). The randomized ENDEAR Phase III study (NCT02929576) comparing enzalutamide and paclitaxel to placebo and paclitaxel in advanced TNBC was in place (100) but withdrawn in 2018, citing that further understanding about the role of androgen signaling in TNBC was required. The TBCRC 032 Phase Ib/II trial (NCT02457910) investigated the safety and efficacy of enzalutamide alone or in combination with $\mathrm{PI} 3 \mathrm{~K}$ inhibitor taselisib in patients with metastatic $\mathrm{AR}^{+} \mathrm{TNBC}$. Primary endpoint of CBR at 16 weeks was $36 \%$ and median PFS was 3.4 months. The trial was not completed due to termination of the development of taselisib. Although this study was exploratory due to sample size limitation, it revealed subtype-specific treatment response (favorable trend for luminal over non-luminal) and identified novel Fgfr2 gene fusions that likely activate the PI3K pathway and AR splice variants that may contribute to enzalutamide resistance. Therefore, an AR IHC score of $\geq 10 \%$ alone may not identify patients with AR-dependent tumors, and LAR subtype and AR splice variants may help identify patients likely to benefit from AR antagonists (101).

\section{Cell surface targets}

Tumor-associated carbohydrate antigens. The Globo $\mathrm{H}$ antigen is a hexasaccharyl sphingolipid expressed on the surface of various cancer types and has been explored as a potential target for vaccine therapy. Adagloxad simolenin (AS) is an immune stimulant comprising the Globo $\mathrm{H}$ hexasaccharide epitope linked to the carrier protein keyhole limpet hemocyanin (KLH). KLH facilitates a more vigorous immune response given the weak antigen, Globo $\mathrm{H}$. As a first-in-class active immunotherapy in development for metastatic breast cancer, AS with the saponin-based adjuvant OBI-821 induced antibodies reactive with Globo $\mathrm{H}^{+}$tumor cells that mediate antibody-dependent cell-mediated cytotoxicity (ADCC) and complement-dependent cytotoxicity (CDC) (102). Phase II trial (NCT01516307) assessed low-dose cyclophosphamide with or without active immunotherapy (AS + adjuvant) in post-treated metastatic breast cancer subjects with stable disease or response to treatment. Although it did not meet its primary efficacy endpoint of PFS, patients who developed an immune response to the vaccine showed significantly improved PFS and OS (103). Based on these subgroup data, Phase III study (NCT03562637) of AS with adjuvant vs. placebo treatment is in progress for high-risk early-stage TNBC patients following neoadjuvant or adjuvant chemotherapy. Patients will be screened for Globo $\mathrm{H}$ expression (IHC H-score $\geq 15$ ) and the primary objective is improvement of invasive disease-free survival (IDFS) in the time frame of 5 years.

Antibody-drug conjugates (ADCs). An ADC is designed to be stable in plasma, target a tumor cell surface antigen with a high affinity and specificity, and is internalized, cleaved, and releases a payload drug which drives antitumor activity through direct cytotoxic cell killing and induces ICD.

Sacituzumab govitecan-hziy (SG) targets a glycoprotein, the human trophoblast cell-surface antigen 2 (TROP-2), that is expressed in more than $90 \%$ of TNBCs. Its payload is the active metabolite of irinotecan (SN-38), which is conjugated to the anti-TROP-2 antibody by a cleavable linker. Phase I/II single group study (NCT01631552) included 108 patients with TNBC and $80 \%$ of patients had visceral metastases. The median number of prior regimens was 3 (range, 2-10), which included chemotherapies and checkpoint inhibitors. Although it did not include biomarker selection of patients, 57 patients had moderate (2+) to strong (3+) and 5 patients had weak or absent TROP-2 expression by IHC according to available data. The ORR was $33 \%$ and the median duration of response (DOR) was 7.7 months. The median PFS was 5.5 months and the median OS was 13.0 months. Myelotoxic effects were the main adverse reactions and grade 3 or 4 AEs included anemia and neutropenia (104). The confirmatory ASCENT Phase III study (NCT02574455) of SG in comparison with treatment of physician's choice for patients with metastatic TNBC was stopped due to compelling evidence of efficacy across multiple endpoints and SG was granted accelerated approval by the FDA based on the results of the IMMU-132-01 Phase II clinical trial for the treatment of adult patients with metastatic TNBC who have received $\geq 2$ prior therapies for metastatic disease. It is the first ADC approved by the FDA specifically for relapsed or refractory metastatic TNBC as well as the first FDA-approved anti-TROP-2 ADC.

Ladiratuzumab vedotin (LV) targets LIV-1, which is expressed in $>90 \%$ of breast tumors with limited expression in normal tissues. LIV-1 is a transmembrane protein with 
zinc transporter and metalloproteinase activity. The payload of LV is the microtubule disrupting agent monomethyl auristatin E (MMAE). Phase I study (NCT01969643) in patients with heavily pretreated metastatic TNBC showed $25 \%$ ORR and medium PFS of 11 weeks. Treatment was generally well-tolerated and related AEs were neutropenia, anemia, and neuropathy (105). LV was further explored in combination studies and in earlier lines of treatment. The SGNLVA-002 Phase Ib/II trial (NCT03310957) was designed to assess whether combining LV and pembrolizumab results in synergistic activity through $\mathrm{LV}$-induced ICD that creates a microenvironment favorable for enhanced anti-PD-L1 activity. It was for first-line treatment of patients with unresectable locally advanced or metastatic TNBC. Initial dose-finding studies revealed ORR of $35 \%$ with responses independent of PD-L1 status and manageable toxicity (106).

ADC has also been explored for HER2-low or negative breast cancer. The rationale is based on the bystander effect, that is, the cleaved drug from an ADC may leak from the targeted tumor cell and affect cells in close proximity regardless of their target antigen expression status. Thus, an ADC having a high drug-to-antibody ratio and high-potency payload would increase the killing of tumor cells even with low HER2 expression. Trastuzumab deruxtecan (T-DXd) is the first HER2-targeted agent to demonstrate promising clinical antitumor activity with a manageable safety profile in patients considered to be HER2-negative. T-DXd delivers a potent topoisomerase I inhibitor payload (an exatecan derivative) which is linked to a humanized anti-HER2 antibody. In Phase Ib (NCT02564900) trial of T-DXd for heavily pretreated patients with advanced HER2-low breast cancer, ORR was $37 \%$ with the median DOR being 10.4 months. Most toxicities were gastrointestinal or hematologic-related, and interstitial lung disease (ILD) was an important identified risk (107). The DESTINY-Breast04 Phase III (NCT03734029) was initiated to compare the efficacy and safety of T-DXd to physician's choice (capecitabine, eribulin, gemcitabine, paclitaxel, or nab-paclitaxel) in patients with HER2-low, unresectable, and/or metastatic breast cancer (108).

\section{New potential therapeutic strategies}

Conversion of TNBC: Access to endocrine therapy. Gene expression analysis and functional studies have revealed a high degree of plasticity and heterogeneity in luminal and basal-like tumors. Expression of ER $\alpha$, FOXA1 or GATA3 can result in transition from basal-like breast cancer to luminal type whereas epigenetic reprogramming can result in a reverse transition (109-111). The CDK2-EZH2 axis in tumors with TNBC phenotype (i.e. basal-like breast cancer) has been explored for conversion to the $\mathrm{ER}^{+}$subtype. Epigenetic enzyme EZH2, a histone-lysine $\mathrm{N}$-methyltransferase that promotes histone $\mathrm{H} 3$ lysine 27 mono-, di- and tri-methylation (H3K27me1/2/3), drives transcriptional repression $(112,113)$. EZH2 can be phosphorylated at T416 (pT416-EZH2) by cyclin E/CDK2 and $>80 \%$ of TNBC patient specimens exhibit high pT416-EZH2 levels, which correlate with poorer survival (114). In preclinical studies, transgenic expression of a phospho-mimicking mutant
EZH2(T416D) in the mammary glands of mice reprogramed the committed luminal breast cancer cells into the basal-like TNBC phenotype. In this setting inhibition of the CDK2-EZH2 axis by EZH2 inhibitors reactivated ER $\alpha$ expression and thus combination with tamoxifen suppressed tumor growth and improved the survival of mice bearing tumors with the TNBC phenotype (115). Therefore, inhibitors of CDK2 or EZH2 combined with hormonal therapy may be a novel therapeutic strategy in TNBC with especially high pT416-EZH2 levels.

Another mechanism-based therapy exploits the lack of ER expression due to hypermethylation of the ER $\alpha$ promoter. A combination epigenetic therapy of a DNA methyltransferase (DNMT) inhibitor and a histone deacetylase (HDAC) inhibitor led to re-expression of genes including $\operatorname{ER} \alpha$ and restored tamoxifen sensitivity in ER-negative breast cancer models $(116,117)$. However, Phase II study (NCT01349959) in patients with advanced hormone-resistant breast cancer or TNBC revealed that combination of DNMT inhibitor 5-azacitidine and HDAC inhibitor entinostat did not induce ER $\alpha$ expression and primary endpoint ORR was not met (118). ER $\alpha$ re-expression induced by DNMT/HDAC inhibition might be attenuated by an active $\mathrm{CDK} 2-\mathrm{EZH} 2$ axis, which affected outcomes in this study.

The conversion of basal-like breast cancer into $\mathrm{ER}^{+}$is also under microenvironmental control. A paracrine signaling network involving platelet-derived growth factor (PDGF)-CC and PDGF receptor- $\alpha$ accelerated tumor growth through recruitment and activation of different subsets of cancer-associated fibroblasts (119). In mouse models, impairing PDGF signaling was found to convert basal-like breast cancers into $\mathrm{ER} \alpha^{+}$, and thus enhanced sensitivity to tamoxifen in previously resistant tumors (120). Therefore, PDGF inhibitors combined with endocrine therapy may be a novel therapeutic strategy in TNBC treatment.

Adaptive clinical studies: Molecular markers. Under the master protocol framework, basket trials, where a targeted therapy is evaluated for multiple diseases that share common molecular alterations, and umbrella trials, where multiple targeted therapies are evaluated for a single disease that is stratified into multiple subgroups based on different molecular factors, have been developed (121). Recently there have been more adaptive, signal-finding clinical trial designs coupled with correlative studies to investigate mechanisms of action. They also facilitate identifying active drug combinations as well as novel tumor indications. Patients are enrolled based on molecular markers from genetic profiling performed on their tumors. Some examples are listed below.

In the OLAPCO Phase II trial (NCT02576444), PARP inhibitor olaparib was assessed in combination with various agents according to identified tumor mutations. It included AKT inhibitor capivasertib for tumors with mutations in the PI3K-AKT pathway, WEE1 inhibitor adavosertib for tumors with tp53 or/and Kras mutations, and ATR inhibitor ceralasertib for tumors with mutations in HRR genes. Primary outcome measure was ORR, and the trial also identified genetic determinants of response and resistance. Another Phase II trial (NCT03718091) evaluated ATR inhibitor M6620 in selected solid tumors. Patients were enrolled in different cohorts based on tumor mutation status, including truncating 
Atm mutations, germline Brca mutations, somatic Brca mutations or other HRR gene mutations, c-MYC amplification, Fbxw7 mutations, cyclin E amplification, and Aridla mutations. Primary outcome measures included disease control rate (DCR) and changes in pCHK1 and $\gamma \mathrm{H} 2 \mathrm{AX}$ levels. The I-SPY 2 Phase II trial (NCT01042379) was a neoadjuvant breast cancer trial using response-adaptive randomization. It had multiple concurrent experimental arms with shared controls. Each biomarker signature was established at trial entry. A new regimen of combination with standard chemotherapy will be moved up to Phase III trial if it shows a high probability of improved pCR over standard chemotherapy.

\section{Conclusion}

Developing novel treatments in both early and advanced TNBC settings remains a significant unmet need. Recent advances with novel agents have been made for specific subgroups with PD-L1 ${ }^{+}$tumors or gBRCAm tumors. However, only a fraction of those patients respond to immune checkpoint or PARP inhibitors, and even those who do respond often develop resistance and relapse. In diverse tumor microenvironments, a given therapeutic agent shows variable responses, thus compromising the survival endpoints especially in an unselected TNBC population. Therefore, developing novel predictive biomarkers are crucial for selecting patients that will benefit the most from a given therapy. Single cell technologies will provide additional insight on tumor-stroma interactions and facilitate compelling rationale for new treatments based on novel biomarkers. A non-invasive testing of plasma circulating tumor DNA (ctDNA) and CTCs can potentially provide real-time disease monitoring and even early therapy modification. However, their prognostic value needs further evaluation. With recent advances in multiomic analyses of cancers, there appears to be genomic and molecular similarities between TNBC and high-grade serous ovarian carcinoma (HGSOC), suggesting that similar biological mechanisms drive some aspects of both cancer types. Therefore, treatment strategies for HGSOC can be explored in TNBC as well. The recent increase in the number of clinical trials investigating various new agents and combination strategies reflects further efforts to understand molecular and immunological aspects of TNBC. This may lead to more meaningful clinical benefits, including event-free and overall survival.

\section{Acknowledgements}

The authors would like to thank Professor Ian Collins of the Institute of Cancer Research, UK for valuable discussions on the DNA damage response pathways and checkpoint kinases.

\section{Funding}

No funding was declared.

\section{Availability of data and materials}

All information provided in this review is documented with relevant and current references.

\section{Authors' contributions}

KAW was responsible for conceptualization, design, interpretation and visualization. KAW and CS were responsible for writing, reviewing and editing. Both authors approved the final manuscript.

\section{Ethics approval and consent to participate}

Not applicable.

\section{Patient consent for publication}

Not applicable.

\section{Competing interests}

No competing interests are declared.

\section{References}

1. Lehmann BD, Bauer JA, Chen X, Sanders ME, Chakravarthy AB, Shyr Y and Pietenpol JA: Identification of human triple-negative breast cancer subtypes and preclinical models for selection of targeted therapies. J Clin Invest 121: 2750-2767, 2011.

2. Lehmann BD, Jovanović B, Chen X, Estrada MV, Johnson KN, Shyr Y, Moses HL, Sanders ME and Pietenpol JA: Refinement of triple-negative breast cancer molecular subtypes: Implications for neoadjuvant chemotherapy selection. PLoS One 11: e0157368, 2016.

3. Giuliano AE, Connolly JL, Edge SB, Mittendorf EA, Rugo HS, Solin LJ, Weaver DL, Winchester DJ and Hortobagyi GN: Breast cancer-major changes in the American Joint Committee on cancer eighth edition cancer staging manual. CA Cancer J Clin 67: 290-303, 2017.

4. Wolff AC, Hammond MEH, Allison KH, Harvey BE, Mangu PB, Bartlett JMS, Bilous M, Ellis IO, Fitzgibbons P, Hanna W, et al: Human epidermal growth factor receptor 2 testing in breast cancer: American Society of Clinical Oncology/College of American pathologists clinical practice guideline focused update. J Clin Oncol 36: 2105-2122, 2018.

5. Allison KH, Hammond MEH, Dowsett M, McKernin SE, Carey LA, Fitzgibbons PL, Hayes DF, Lakhani SR, Chavez-MacGregor M, Perlmutter J, et al: Estrogen and progesterone receptor testing in breast cancer: ASCO/CAP guideline update. J Clin Oncol 38: 1346-1366, 2020.

6. Yam C, Mani SA and Moulder SL: Targeting the molecular subtypes of triple negative breast cancer: Understanding the diversity to progress the field. Oncologist 22: 1086-1093, 2017.

7. Bonotto M, Gerratana L, Poletto E, Driol P, Giangreco M, Russo S, Minisini AM, Andreetta C, Mansutti M, Pisa FE, et al: Measures of outcome in metastatic breast cancer: Insights from a real-world scenario. Oncologist 19: 608-615, 2014.

8. Kohler BA, Sherman RL, Howlader N, Jemal A, Ryerson AB, Henry KA, Boscoe FP, Cronin KA, Lake A, Noone AM, et al: Annual report to the nation on the status of cancer, 1975-2011, featuring incidence of breast cancer subtypes by race/ethnicity, poverty, and state. J Natl Cancer Inst 107: djv048, 2015.

9. O'Shaughnessy J, Schwartzberg L, Danso MA, Miller KD, Rugo HS, Neubauer M, Robert N, Hellerstedt B, Saleh M, Richards P, et al: Phase III study of iniparib plus gemcitabine and carboplatin versus gemcitabine and carboplatin in patients with metastatic triple-negative breast cancer. J Clin Oncol 32: 3840-3847, 2014.

10. Caswell-Jin JL, Plevritis SK, Tian L, Cadham CJ, Xu C, Stout NK, Sledge GW, Mandelblatt JS and Kurian AW: Change in survival in metastatic breast cancer with treatment advances: Meta-analysis and systematic review. JNCI Cancer Spectr 2: pky062, 2018.

11. Plevritis SK, Munoz D, Kurian AW, Stout NK, Alagoz O, Near AM, Lee SJ, van den Broek JJ, Huang X, Schechter CB, et al: Association of screening and treatment with breast cancer mortality by molecular subtype in US women, 2000-2012. JAMA 319: 154-164, 2018. 
12. Stanton SE, Adams S and Disis ML: Variation in the incidence and magnitude of tumor-infiltrating lymphocytes in breast cancer subtypes: A systematic review. JAMA Oncol 2: 1354-1360, 2016.

13. Safonov A, Jiang T, Bianchini G, Győrffy B, Karn T, Hatzis C and Pusztai L: Immune gene expression is associated with genomic aberrations in breast cancer. Cancer Res 77: 3317-3324, 2017.

14. Schmid P, Adams S, Rugo HS, Schneeweiss A, Barrios $\mathrm{CH}$ Iwata H, Diéras V, Hegg R, Im SA, Shaw Wright G, et al: Atezolizumab and Nab-Paclitaxel in advanced triple-negative breast cancer. N Engl J Med 379: 2108-2121, 2018.

15. Schmid P, Rugo HS, Adams S, Schneeweiss A, Barrios $\mathrm{CH}$, Iwata H, Diéras V, Henschel V, Molinero L, Chui SY, et al: Atezolizumab plus nab-paclitaxel as first-line treatment for unresectable, locally advanced or metastatic triple-negative breast cancer (IMpassion130): Updated efficacy results from a randomised, double-blind, placebo-controlled, phase 3 trial. Lancet Oncol 21: 44-59, 2020.

16. Dudley JC, Lin MT, Le DT and Eshleman JR: Microsatellite instability as a biomarker for PD-1 blockade. Clin Cancer Res 22: 813-820, 2016

17. Bonneville R, Krook MA, Kautto EA, Miya J, Wing MR, Chen HZ, Reeser JW, Yu L and Roychowdhury S: Landscape of microsatellite instability across 39 cancer types. JCO Precis Oncol 2017: PO.17.00073, 2017.

18. Kurata K, Kubo M, Mori H, Kawaji H, Motoyama Y, Kuroki L, Yamada M, Kaneshiro K, Kai M and Nakamura M: Microsatellite instability in triple negative breast cancers. In: Proceedings of the 2018 San Antonio Breast Cancer Symposium. Cancer Res 79 (Suppl 4): Abstract nr P1-06-11. 2019.

19. Bryant HE, Schultz N, Thomas HD, Parker KM, Flower D, Lopez E, Kyle S, Meuth M, Curtin NJ and Helleday T: Specific killing of BRCA2-deficient tumours with inhibitors of poly(ADP-ribose) polymerase. Nature 434: 913-917, 2005.

20. Farmer H, McCabe N, Lord CJ, Tutt AN, Johnson DA, Richardson TB, Santarosa M, Dillon KJ, Hicks on I, Knights C, et al: Targeting the DNA repair defect in BRCA mutant cells as a therapeutic strategy. Nature 434: 917-921, 2005

21. Robson M, Im SA, Senkus E, Xu B, Domchek SM, Masuda N, Delaloge S, Li W, Tung N, Armstrong A, et al: Olaparib for metastatic breast cancer in patients with a germline BRCA mutation. N Engl J Med 377: 523-533, 2017.

22. Robson ME, Tung N, Conte P, Im SA, Senkus E, Xu B, Masuda N Delaloge S, Li W, Armstrong A, et al: OlympiAD final overall survival and tolerability results: Olaparib versus chemotherapy treatment of physician's choice in patients with a germline BRCA mutation and HER2-negative metastatic breast cancer. Ann Oncol 30: 558-566, 2019.

23. Litton JK, Rugo HS, Ettl J, Hurvitz SA, Gonçalves A, Lee KH, Fehrenbacher L, Yerushalmi R, Mina LA, Martin M, et al: Talazoparib in patients with advanced breast cancer and a germline BRCA mutation. N Engl J Med 379: 753-763, 2018.

24. Denkert C, von Minckwitz G, Darb-Esfahani S, Lederer B, Heppner BI, Weber KE, Budczies J, Huober J, Klauschen F, Furlanetto J, et al: Tumour-infiltrating lymphocytes and prognosis in different subtypes of breast cancer: A pooled analysis of 3771 patients treated with neoadjuvant therapy. Lancet Oncol 19: 40-50, 2018.

25. Hida AI, Watanabe T, Sagara Y, Kashiwaba M, Sagara Y, Aogi K, Ohi Y and Tanimoto A: Diffuse distribution of tumor-infiltrating lymphocytes is a marker for better prognosis and chemotherapeutic effect in triple-negative breast cancer. Breast Cancer Res Treat 178: 283-294, 2019.

26. Loi S, Drubay D, Adams S, Pruneri G, Francis PA, Lacroix-Triki M, Joensuu H, Dieci MV, Badve S, Demaria S, et al: Tumor-infiltrating lymphocytes and prognosis: A pooled individual patient analysis of early-stage triple-negative breast cancers. J Clin Oncol 37: 559-569, 2019.

27. Galon J and Bruni D: Approaches to treat immune hot, altered and cold tumours with combination immunotherapies. Nat Rev Drug Discov 18: 197-218, 2019.

28. Adams S, Gatti-Mays ME, Kalinsky K, Korde LA, Sharon E, Amiri-Kordestani L, Bear H, McArthur HL, Frank E, Perlmutter J, et al: Current landscape of immunotherapy in breast cancer: A review. JAMA Oncol: Apr 11, 2019 (Epub ahead of print).

29. Schmid P, Salgado R, Park YH, Muñoz-Couselo E, Kim SB Sohn J, Im S-A, Foukakis T, Kuemmel S, Dent R, et al: Pembrolizumab plus chemotherapy as neoadjuvant treatment of high-risk, early-stage triple-negative breast cancer: Results from the phase $1 \mathrm{~b}$ open-label, multicohort KEYNOTE-173 study. Ann Oncol 31: 569-581, 2020
30. Nanda R, Liu MC, Yau C, Shatsky R, Pusztai L, Wallace A, Chien AJ, Forero-Torres A, Ellis E, Han H, et al: Effect of pembrolizumab plus neoadjuvant chemotherapy on Pathologic complete response in women with early-stage breast cancer: An analysis of the ongoing phase 2 adaptively randomized I-SPY2 trial. JAMA Oncol 6: 1-9, 2020.

31. Schmid P, Cortes J, Pusztai L, McArthur H, Kümmel S, Bergh J, Denkert C, Park YH, Hui R, Harbeck N, et al: Pembrolizumab for early triple-negative breast cancer. N Engl J Med 382: 810-821, 2020.

32. Gianni L, Huang CS, Egle D, Bermejo B, Zamagni C, Thill M, Anton A, Zambelli S, Bianchini G, Russo S and Ciruelos E: Pathologic complete response (pCR) to neoadjvaunt treatment with or without atezolizumab in triple negative, early high-risk and locally advanced breast cancer. NeoTRIPaPDL1 Michelangelo randomized study. In: Proceedings of the 2019 San Antonio Breast Cancer Symposium. Cancer Res 80 (Suppl 4): Abstract nr GS3-04, 2020.

33. Mittendorf E, Barrios CH, Harbeck N, Miles D, Saji S, Zhang H, Duc AN, Rafii S and Lai C: IMpassion031: A phase III study comparing neoadjuvant atezolizumab vs placebo in combination with nab-paclitaxel-based chemotherapy in early triple-negative breast cancer (TNBC). In: Proceedings of the 2017 San Antonio Breast Cancer Symposium. Cancer Res 78 (Suppl 4): Abstract nr OT2-07-03, 2018.

34. Cortes J, Cescon DW, Rugo HS, Nowecki Z, Im SA, Yusof MM, Gallardo C, Lipatov O, Barrios CH, Holgado E, et al: KEYNOTE-355: Randomized, double-blind, phase III study of pembrolizumab + chemotherapy versus placebo + chemotherapy for previously untreated locally recurrent inoperable or metastatic triple-negative breast cancer. J Clin Oncol 38: (Suppl 15): S1000-S1000, 2020.

35. Voorwerk L, Slagter M, Horlings HM, Sikorska K, van de Vijver KK, de Maaker M, Nederlof I, Kluin RJC, Warren S, Ong S, et al: Immune induction strategies in metastatic triple-negative breast cancer to enhance the sensitivity to PD-1 blockade: The TONIC trial. Nat Med 25: 920-928, 2019.

36. Allard B, Longhi MS, Robson SC and Stagg J: The ectonucleotidases CD39 and CD73: Novel checkpoint inhibitor targets. Immunol Rev 276: 121-144, 2017.

37. Ghalamfarsa G, Kazemi MH, Raoofi Mohseni S, Masjedi A, Hojjat-Farsangi M, Azizi G, Yousefi M and Jadidi-Niaragh F: CD73 as a potential opportunity for cancer immunotherapy. Expert Opin Ther Targets 23: 127-142, 2019.

38. Duhant X, Schandené L, Bruyns C, Gonzalez NS, Goldman M, Boeynaems JM and Communi D: Extracellular adenine nucleotides inhibit the activation of human CD4+ T lymphocytes. J Immunol 169: 15-21, 2002.

39. Allard B, Beavis PA, Darcy PK and Stagg J: Immunosuppressive activities of adenosine in cancer. Curr Opin Pharmacol 29: 7-16, 2016.

40. Ohta A: A metabolic immune checkpoint: Adenosine in tumor microenvironment. Front Immunol 7: 109, 2016.

41. Buisseret L, Pommey S, Allard B, Garaud S, Bergeron M, Cousineau I, Ameye L, Bareche Y, Paesmans M, Crown JPA, et al: Clinical significance of CD73 in triple-negative breast cancer: Multiplex analysis of a phase III clinical trial. Ann Oncol 29: 1056-1062, 2018

42. Powderly J, Spira A, Gutierrez R, DiRenzo D, Udyavar A, Karakunnel JJ, Rieger A, Colabella J, Lai DW and de Souza P: Phase 1 evaluation of AB928, a novel dual adenosine receptor antagonist, combined with chemotherapy or AB122 (anti-PD-1) in patients with advanced malignancies. Ann Oncol 30 (Suppl 5): v475-v532, 2019

43. Hartman AR, Kaldate RR, Sailer LM, Painter L, Grier CE, Endsley RR, Griffin M, Hamilton SA, Frye CA, Silberman MA, et al: Prevalence of BRCA mutations in an unselected population of triple-negative breast cancer. Cancer 118: 2787-2795, 2012

44. Okuma HS and Yonemori K: BRCA gene mutations and poly(ADP-Ribose) polymerase inhibitors in triple-negative breast cancer. Adv Exp Med Biol 1026: 271-286, 2017.

45. Lord CJ and Ashworth A: BRCAness revisited. Nat Rev Cancer 16: 110-120, 2016

46. Litton JK, Scoggins ME, Hess KR, Adrada BE, Murthy RK, Damodaran S, DeSnyder SM, Brewster AM, Barcenas CH, Valero V, et al: Neoadjuvant talazoparib for patients with operable breast cancer with a germline BRCA pathogenic variant. J Clin Oncol 38: 388-394, 2020. 
47. Loibl S, O'Shaughnessy J, Untch M, Sikov WM, Rugo HS, McKee MD, Huober J, Golshan M, von Minckwitz G, Maag D, et al: Addition of the PARP inhibitor veliparib plus carboplatin or carboplatin alone to standard neoadjuvant chemotherapy in triple-negative breast cancer (BrighTNess): A randomised, phase 3 trial. Lancet Oncol 19: 497-509, 2018.

48. Jiao S, Xia W, Yamaguchi H, Wei Y, Chen MK, Hsu JM, Hsu JL, Yu WH, Du Y, Lee HH, et al: PARP inhibitor upregulates PD-L1 expression and enhances cancer-associated immunosuppression. Clin Cancer Res 23: 3711-3720, 2017.

49. Vinayak S, Tolaney SM, Schwartzberg L, Mita M, McCann G, Tan AR, Wahner-Hendrickson AE, Forero A, Anders C, Wulf GM, et al: Open-label clinical trial of niraparib combined with pembrolizumab for treatment of advanced or metastatic triple-negative breast cancer. JAMA Oncol 5: 1132-1140, 2019.

50. Domchek S, Postel-Vinay S, Im S, Park YH, Delord J, Italiano A, Alexandre J, You B, Bastian S, Krebs MG, et al: Phase II study of olaparib (o) and durvalumab (d) (MEDIOLA): Updated results in patients (pts) with germline BRCA-mutated (gBRCAm) metastatic breast cancer (mbc). Ann Oncol 30 (Suppl 5): v475-v532, 2019.

51. Pusztai L, Han HS, Yau C, Wolf D, Wallace AM, Shatsky R, Helsten T, Boughey JC, Haddad T, Stringer-Reasor E, et al Durvalumab in combination with olaparib and paclitaxel in high-risk HER2 negative stage II/III breast cancer: Results from the I-SPY 2 trial. In: Proceedings of the Annual Meeting of the American Association for Cancer Research 2020. Cancer Res 80 (Suppl 16): Abstract nr CT011, 2020.

52. Mitri ZI, Vuky J, Kemmer KA, Savin MA, Parmar S, Kolodzie AK, Johnson B, Williams-Belizaire R, Gray JW and Mills GB: A phase II trial of olaparib and durvalumab in metastatic BRCA wild type triple-negative breast cancer. J Clin Oncol 37: TPS1111, 2019.

53. Rugo HS, Llombart-Cussac A, Andre F, Robson ME, Saji S, Harbeck N, Schmid P, Cescon DW, Ahn JS, Nanda R, et al: KEYLYNK-009: A phase II/III, open-label, randomized study of pembrolizumab (pembro) plus olaparib vs pembro plus chemotherapy after induction with first-line pembro plus chemotherapy in patients with locally recurrent inoperable or metastatic triple-negative breast cancer (TNBC). J Clin Oncol 38: TPS596, 2020 .

54. Maacke H, Opitz S, Jost K, Hamdorf W, Henning W, Krüger S, Feller AC, Lopens A, Diedrich K, Schwinger E and Stürzbecher HW: Over-expression of wild-type Rad51 correlates with histological grading of invasive ductal breast cancer. Int J Cancer 88: 907-913, 2000.

55. Martin RW, Orelli BJ, Yamazoe M, Minn AJ, Takeda S and Bishop DK: RAD51 up-regulation bypasses BRCA1 function and is a common feature of BRCA1-deficient breast tumors. Cancer Res 67: 9658-9665, 2007.

56. Wiegmans AP, Yap PY, Ward A, Lim YC and Khanna KK: Differences in expression of key DNA damage repair genes after epigenetic-induced BRCAness dictate synthetic lethality with PARP1 inhibition. Mol Cancer Ther 14: 2321-2331, 2015.

57. Liu Y, Burness ML, Martin-Trevino R, Guy J, Bai S, Harouaka R, Brooks MD, Shang L, Fox A, Luther TK, et al: RAD51 mediates resistance of cancer stem cells to PARP inhibition in triple-negative breast cancer. Clin Cancer Res 23: 514-522, 2017.

58. Marzio A, Puccini J, Kwon Y, Maverakis NK, Arbini A, Sung P, Bar-Sagi D and Pagano M: The F-Box domain-dependent activity of EMI1 regulates PARPi sensitivity in triple-negative breast cancers. Mol Cell 73: 224-237.e6, 2019.

59. Tutt A, Stephens C, Frewer P, Pierce A, Rhee J, So K, Ottesen L, Dean E and Hollingsworth SJ: VIOLETTE: A randomized phase II study to assess DNA damage response inhibitors in combination with olaparib (Ola) vs. Ola monotherapy in patients (pts) with metastatic, triple-negative breast cancer (TNBC) stratified by alterations in homologous recombination repair (HRR)-related genes. J Clin Oncol 36 (Suppl 15): TPS1112, 2018

60. Hirai H, Arai T, Okada M, Nishibata T, Kobayashi M, Sakai N, Imagaki K, Ohtani J, Sakai T, Yoshizumi T, et al: MK-1775, a small molecule Weel inhibitor, enhances anti-tumor efficacy of various DNA-damaging agents, including 5-fluorouracil. Cancer Biol Ther 9: 514-522, 2010.

61. Pitts TM, Simmons DM, Bagby SM, Hartman SJ, Yacob BW, Gittleman B, Tentler JJ, Cittelly D, Ormond DR, Messersmith WA, et al: Wee1 inhibition enhances the anti-tumor effects of capecitabine in preclinical models of triple-negative breast cancer. Cancers (Basel) 12: 719, 2020
62. Do K, Wilsker D, Ji J, Zlott J, Freshwater T, Kinders RJ, Collins J, Chen AP, Doroshow JH and Kummar S: Phase I study of single-agent AZD1775 (MK-1775), a Wee1 kinase inhibitor, in patients with refractory solid tumors. J Clin Oncol 33: 3409-3415, 2015

63. Do KT, Hill SJ, Kochupurakkal B, Supko JG, Gannon C, Anderson A, Muzikansky A, Wolanski A, Hedglin J, Parmar K, et al: Abstract CT232: Phase I combination study of the CHK1 inhibitor prexasertib (LY2606368) and olaparib in patients with high-grade serous ovarian cancer and other advanced solid tumors. In: Proceedings of the American Association for Cancer Research Annual Meeting 2019. Cancer Res 79 (Suppl 13): Abstract nr CT232, 2019.

64. Peng W, Chen JQ, Liu C, Malu S, Creasy C, Tetzlaff MT, Xu C, McKenzie JA, Zhang C, Liang X, et al: Loss of PTEN promotes resistance to T cell-mediated immunotherapy. Cancer Discov 6: 202-216, 2016

65. André F, Ciruelos E, Rubovszky G, Campone M, Loibl S, Rugo HS, Iwata H, Conte P, Mayer IA, Kaufman B, et al: Alpelisib for PIK3CA-mutated, hormone receptor-positive advanced breast cancer. N Engl J Med 380: 1929-1940, 2019.

66. Kim SB, Dent R, Im SA, Espié M, Blau S, Tan AR, Isakoff SJ, Oliveira M, Saura C, Wongchenko MJ, et al: Ipatasertib plus paclitaxel versus placebo plus paclitaxel as first-line therapy for metastatic triple-negative breast cancer (LOTUS): A multicentre, randomised, double-blind, placebo-controlled, phase 2 trial. Lancet Oncol 18: 1360-1372, 2017.

67. Dent R, Im SA, Espie M, Blau S, Tan AR, Isakoff SJ, Oliveira M, Saura C, Wongchenko M, Kapp AV, et al: Overall survival (OS) update of the double-blind placebo (PBO)-controlled randomized phase 2 LOTUS trial of first-line ipatasertib (IPAT) + paclitaxel (PAC) for locally advanced/metastatic triple-negative breast cancer (mTNBC). J Clin Oncol 36: 1008, 2018.

68. Dent R, Kim SB, Oliveira M, Isakoff SJ, Barrios $\mathrm{CH}$, O'Shaughnessy J, Lu X, Wongchenko M, Bradley D, Mani A, et al: IPATunity130: A pivotal randomized phase III trial evaluating ipatasertib (IPAT) + paclitaxel (PAC) for PIK3CA/AKT1/PTEN-altered advanced triple-negative (TN) or hormone receptor-positive HER2-negative (HR+/HER2-) breast cancer (BC). J Clin Oncol 36 (Suppl 15): TPS1117, 2018.

69. Schmid P, Abraham J, Chan S, Wheatley D, Brunt AM, Nemsadze G, Baird RD, Park YH, Hall PS, Perren T, et al: Capivasertib plus paclitaxel versus placebo plus paclitaxel as first-line therapy for metastatic triple-negative breast cancer: The PAKT trial. J Clin Oncol 38: 423-433, 2020.

70. Schmid P, Cortes J, Robson M, Iwata H, Hegg R, Verma S, Nechaeva M, Xu B, Haddad V, Imedio RE, et al: Abstract OT2-08-02: Capivasertib and paclitaxel in first-line treatment of patients with metastatic triple-negative breast cancer: A phase III trial (CAPItello-290). In: Proceedings of the 2019 San Antonio Breast Cancer Symposium. Cancer Res 80 (Suppl 4): Abstract nr OT2-08-02, 2020.

71. Schmid P, Loirat D, Savas P, Espinosa E, Boni V, Italiano A, White S, Singel MS, Withana N, Mani A, et al: Phase Ib study evaluating a triplet combination of ipatasertib (IPAT), atezolizumab (atezo), and paclitaxel (PAC) or nab-PAC as first-line (1L) therapy for locally advanced/metastatic triple-negative breast cancer (TNBC). In: Proceedings of the American Association for Cancer Research Annual Meeting 2019. Cancer Res 79 (Suppl 13): Abstract nr CT049, 2019.

72. Hanahan D and Weinberg RA: Hallmarks of cancer: The next generation. Cell 144: 646-674, 2011.

73. Dean JL, McClendon AK and Knudsen ES: Modification of the DNA damage response by therapeutic CDK4/6 inhibition. J Biol Chem 287: 29075-29087, 2012.

74. Cretella D, Fumarola C, Bonelli M, Alfieri R, La Monica S, Digiacomo G, Cavazzoni A, Galetti M, Generali D and Petronini PG: Pre-treatment with the CDK4/6 inhibitor palbociclib improves the efficacy of paclitaxel in TNBC cells. Sci Rep 9: 13014, 2019.

75. Clark AS, McAndrew NP, Troxel A, Feldman M, Lal P, Rosen M, Burrell J, Redlinger C, Gallagher M, Bradbury AR, et al: Combination paclitaxel and palbociclib: Results of a phase I trial in advanced breast cancer. Clin Cancer Res 25: 2072-2079, 2019.

76. Tan AR, Wright GS, Thummala AR, Danso MA, Popovic L, Pluard TJ, Han HS, Vojnović Ž, Vasev N, Ma L, et al: Trilaciclib plus chemotherapy versus chemotherapy alone in patients with metastatic triple-negative breast cancer: A multicentre, randomised, open-label, phase 2 trial. Lancet Oncol 20: 1587-1601, 2019.

77. Stine ZE, Walton ZE, Altman BJ, Hsieh AL and Dang CV: MYC, metabolism, and cancer. Cancer Discov 5: 1024-1039, 2015. 
78. Ambrosio S, Amente S, Napolitano G, Di Palo G, Lania L and Majello B: MYC impairs resolution of site-specific DNA double-strand breaks repair. Mutat Res 774: 6-13, 2015.

79. Wiegmans AP, Al-Ejeh F, Chee N, Yap PY, Gorski JJ, Da Silva L, Bolderson E, Chenevix-Trench G, Anderson R, Simpson PT, et al: Rad51 supports triple negative breast cancer metastasis. Oncotarget 5: 3261-3272, 2014.

80. Carey JPW, Karakas C, Bui T, Chen X, Vijayaraghavan S, Zhao Y, Wang J, Mikule K, Litton JK, Hunt KK and Keyomarsi K: Synthetic lethality of PARP inhibitors in combination with MYC blockade is independent of BRCA status in triple negative breast cancer. Cancer Res 78: 742-757, 2018.

81. Horiuchi D, Kusdra L, Huskey NE, Chandriani S, Lenburg ME, Gonzalez-Angulo AM, Creasman KJ, Bazarov AV, Smyth JW, Davis SE, et al: MYC pathway activation in triple-negative breast cancer is synthetic lethal with CDK inhibition. J Exp Med 209: 679-696, 2012.

82. Hossain DMS, Javaid S, Cai M, Zhang C, Sawant A, Hinton M, Sathe M, Grein J, Blumenschein W, Pinheiro EM and Chackerian A: Dinaciclib induces immunogenic cell death and enhances anti-PD1-mediated tumor suppression. J Clin Invest 128: 644-654, 2018.

83. Chien AJ, Gliwa AS, Rahmaputri S, Dittrich HF, Majure MC, Rugo HS, Melisko ME, Munster PN, Park JW, Moasser MM, et al: A phase Ib trial of the cyclin-dependent kinase inhibitor dinaciclib (dina) in combination with pembrolizumab $(\mathrm{P})$ in patients with advanced triple-negative breast cancer (TNBC) and response correlation with MYC-overexpression. J Clin Oncol 38: 1076,2020

84. Kono M, Fujii T, Lim B, Karuturi MS, Tripathy D and Ueno NT: Androgen receptor function and androgen receptor-targeted therapies in breast cancer: A Review. JAMA Oncol 3: 1266-1273, 2017.

85. Gerratana L, Basile D, Buono G, De Placido S, Giuliano M, Minichillo S, Coinu A, Martorana F, De Santo I, Del Mastro L, et al: Androgen receptor in triple negative breast cancer: A potential target for the targetless subtype. Cancer Treat Rev 68: 102-110, 2018.

86. Anestis A, Zoi I, Papavassiliou AG and Karamouzis MV: Androgen receptor in breast cancer-clinical and preclinical research insights. Molecules 25: 358, 2020

87. MasudaH,Baggerly KA, Wang Y,Zhang Y,Gonzalez-AnguloAM, Meric-Bernstam F, Valero V, Lehmann BD, Pietenpol JA, Hortobagyi GN, et al: Differential response to neoadjuvant chemotherapy among 7 triple-negative breast cancer molecular subtypes. Clin Cancer Res 19: 5533-5540, 2013.

88. Thike AA, Yong-Zheng Chong L, Cheok PY, Li HH, Wai-Cheong Yip G, Huat Bay B, Tse GM, Iqbal J and Tan PH: Loss of androgen receptor expression predicts early recurrence in triple-negative and basal-like breast cancer. Mod Pathol 27: 352-360, 2014.

89. Echavarria I, Lopez-Tarruella S, Picornell A, García-Saenz JA, Jerez Y, Hoadley K, Gómez HL, Moreno F, Monte-Millan MD, Márquez-Rodas I, et al: Pathological response in a triple-negative breast cancer cohort treated with neoadjuvant carboplatin and docetaxel according to Lehmann's refined classification. Clin Cancer Res 24: 1845-1852, 2018.

90. Santonja A, Sánchez-Muñoz A, Lluch A, Chica-Parrado MR, Albanell J, Chacón JI, Antolín S, Jerez JM, de la Haba J, de Luque V, et al: Triple negative breast cancer subtypes and pathologic complete response rate to neoadjuvant chemotherapy. Oncotarget 9: 26406-26416, 2018.

91. Venema CM, Bense RD, Steenbruggen TG, Nienhuis HH, Qiu SQ, van Kruchten M, Brown M, Tamimi RM, Hospers GAP, Schröder CP, et al: Consideration of breast cancer subtype in targeting the androgen receptor. Pharmacol Ther 200: 135-147, 2019.

92. Rice MA, Malhotra SV and Stoyanova T: Second-generation antiandrogens: From discovery to standard of care in castration resistant prostate cancer. Front Oncol 9: 801, 2019.

93. Gucalp A, Tolaney S, Isakoff SJ, Ingle JN, Liu MC, Carey LA, Blackwell K, Rugo H, Nabell L, Forero A, et al: Phase II trial of bicalutamide in patients with androgen receptor-positive, estrogen receptor-negative metastatic breast cancer. Clin Cancer Res 19: 5505-5512, 2013.

94. Gucalp A, Edelweiss M, Patil S, Gounder MM, Feigin KN, Corben A, Arumov A and Traina TA: Abstract P3-11-04 Phase I/II trial of palbociclib in combination with bicalutamide for the treatment of androgen receptor (AR)+ metastatic breast cancer (MBC). In: Proceedings of the 2017 San Antonio Breast Cancer Symposium. Cancer Res 2018;78 (Suppl 4): Abstract nr P3-11-04, 2018.
95. Gucalp A, Boyle LA, Alano T, Arumov A, Gounder MM, Patil S, Feigin K, Edelweiss M, D'Andrea G, Bromberg J, et al: Phase II trial of bicalutamide in combination with palbociclib for the treatment of androgen receptor $(+)$ metastatic breast cancer. J Clin Oncol 38, 2020.

96. Bonnefoi H, Grellety T, Tredan O, Saghatchian M, Dalenc F, Mailliez A, L'Haridon T, Cottu P, Abadie-Lacourtoisie S, You B, et al: A phase II trial of abiraterone acetate plus prednisone in patients with triple-negative androgen receptor positive locally advanced or metastatic breast cancer (UCBG 12-1). Ann Oncol 27: 812-818, 2016.

97. Gucalp A, Danso MA, Elias AD, Bardia A, Ali HY, Potter D, Gabrail NY, Haley BB, Khong HT, Riley EC, et al: Phase (Ph) 2 stage 1 clinical activity of seviteronel, a selective CYP17-lyase and androgen receptor (AR) inhibitor, in women with advanced AR+ triple-negative breast cancer (TNBC) or estrogen receptor (ER)+ BC: CLARITY-01. J Clin Oncol 35: $1102,2017$.

98. Bardia A, Gucalp A, DaCosta N, Gabrail N, Danso M, Ali H, Blackwell KL, Carey LA, Eisner JR, Baskin-Bey ES and Traina TA: Phase 1 study of seviteronel, a selective CYP17 lyase and androgen receptor inhibitor, in women with estrogen receptor-positive or triple-negative breast cancer. Breast Cancer Res Treat. 171: 111-120, 2018.

99. Traina TA, Miller K, Yardley DA, Eakle J, Schwartzberg LS, O'Shaughnessy J, Gradishar W, Schmid P, Winer E, Kelly C, et al: Enzalutamide for the treatment of androgen receptor-expressing triple-negative breast cancer. J Clin Oncol 36: 884-890, 2018.

100. Dent R, Schmid P, Cortes J, Kim SB, Andre F, Abramson V, Cardoso F, Colleoni M, Morris P, Steinberg J, et al: Abstract OT3-02-02: ENDEAR: A randomized international phase 3 study comparing the efficacy and safety of enzalutamide in combination with paclitaxel chemotherapy or as monotherapy vs placebo with paclitaxel in patients with advanced diagnostic-positive triple-negative breast cancer. Cancer Res 77: Abstract OT3-02-02, 2017.

101. Lehmann BD, Abramson VG, Sanders ME, Mayer EL, Haddad TC, Nanda R, Van Poznak C, Storniolo AM, Nangia JR, Gonzalez-Ericsson PI, et al: TBCRC 032 IB/II multicenter study: Molecular insights to AR antagonist and PI3K inhibitor efficacy in patients with $\mathrm{AR}^{+}$metastatic triple-negative breast cancer. Clin Cancer Res 26: 2111-2123, 2020.

102. Gilewski T, Ragupathi G, Bhuta S, Williams LJ, Musselli C, Zhang XF, Bornmann WG, Spassova M, Bencsath KP, Panageas KS, et al: Immunization of metastatic breast cancer patients with a fully synthetic globo $\mathrm{H}$ conjugate: A phase I trial. Proc Natl Acad Sci USA 98: 3270-3275, 2001.

103. Huang CS, Yu AL, Tseng LM, Chow LWC, Hou MF, Hurvitz SA, Schwab RB, Wong CH, Murray JL, Chang SC, et al: Randomized phase II/III trial of active immunotherapy with OPT-822/OPT-821 in patients with metastatic breast cancer. J Clin Oncol 34 (Suppl 15): S1003, 2016.

104. Bardia A, Mayer IA, Vahdat LT, Tolaney SM, Isakoff SJ, Diamond JR, O'Shaughnessy J, Moroose RL, Santin AD, Abramson VG, et al: Sacituzumab govitecan-hziy in refractory metastatic triple-negative breast cancer. N Eng J Med 380: 741-751, 2019.

105. Modi S, Pusztai L, Forero A, Mita M, Miller KD, Weise A, Burris H III, Kalinsky K, Tsai M, Liu MC, et al: Abstract PD3-14: Phase 1 study of the antibody-drug conjugate SGN-LIV1A in patients with heavily pretreated triple-negative metastatic breast cancer. Cancer Res 78, 2018.

106. Han HS, Alemany CA, Brown-Glaberman UA, Pluard TJ, Sinha R, Sterrenberg D, Albain KS, Basho RK, Biggs D, Boni V, et al: SGNLVA-002: Single-arm, open label phase $\mathrm{Ib} / \mathrm{II}$ study of ladiratuzumab vedotin (LV) in combination with pembrolizumab for first-line treatment of patients with unresectable locally advanced or metastatic triple-negative breast cancer. J Clin Oncol 37 (Suppl 15): TPS1110, 2019.

107. Modi S, Park H, Murthy RK, Iwata H, Tamura K, Tsurutani J, Moreno-Aspitia A, Doi T, Sagara Y, Redfern C, et al: Antitumor activity and safety of trastuzumab Deruxtecan in patients with HER2-low-expressing advanced breast cancer: Results from a phase Ib study. J Clin Oncol 38: 1887-1896, 2020.

108. Modi S, Ohtani S, Lee CC, Wang K, Saxena K and Cameron DA: A phase III, multicenter, randomized, open label trial of [fam-] trastuzumab deruxtecan (DS-8201a) versus investigator's choice in HER2-low breast cancer. J Clin Oncol 37: (Suppl 15): TPS1102, 2019. 
109. Molyneux G, Geyer FC, Magnay FA, McCarthy A, Kendrick H, Natrajan R, Mackay A, Grigoriadis A, Tutt A, Ashworth A, et al: BRCA1 basal-like breast cancers originate from luminal epithelial progenitors and not from basal stem cells. Cell Stem Cell 7: 403-417, 2010

110. Bernardo GM, Bebek G, Ginther CL, Sizemore ST, Lozada KL, Miedler JD, Anderson LA, Godwin AK, Abdul-Karim FW, Slamon DJ and Keri RA: FOXA1 represses the molecular phenotype of basal breast cancer cells. Oncogene 32: 554-563, 2013

111. Su Y, Subedee A, Bloushtain-Qimron N, Savova V, Krzystanek M, Li L, Marusyk A, Tabassum DP, Zak A, Flacker MJ, et al: Somatic cell fusions reveal extensive heterogeneity in basal-like breast cancer. Cell Rep 11: 1549-1563, 2015.

112. Cao R, Wang L, Wang H, Xia L, Erdjument-Bromage H, Tempst P, Jones RS and Zhang Y: Role of histone H3 lysine 27 methylation in Polycomb-group silencing. Science 298: 1039-1043, 2002.

113. Yamagishi $\mathrm{M}$ and Uchimaru K: Targeting EZH2 in Cancer Therapy. Curr Opin Oncol 29: 375-381, 2017.

114. Yang CC, LaBaff A, Wei Y, Nie L, Xia W, Huo L, Yamaguchi H, Hsu YH, Hsu JL, Liu D, et al: Phosphorylation of EZH2 at T416 by CDK 2 contributes to the malignancy of triple negative breast cancers. Am J Transl Res 7: 1009-1020, 2015.

115. Nie L, Wei Y, Zhang F, Hsu YH, Chan LC, Xia W, Ke B, Zhu C, Deng R, Tang J, et al: CDK2-mediated site-specific phosphorylation of EZH2 drives and maintains triple-negative breast cancer. Nat Commun 10: 5114, 2019.

116. Yang X, Phillips DL, Ferguson AT, Nelson WG, Herman JG and Davidson NE: Synergistic activation of functional estrogen receptor (ER)-alpha by DNA methyltransferase and histone deacetylase inhibition in human ER-alpha-negative breast cancer cells. Cancer Res 61: 7025-7029, 2001
117. Sharma D, Saxena NK, Davidson NE and Vertino PM: Restoration of tamoxifen sensitivity in estrogen receptor-negative breast cancer cells: Tamoxifen-bound reactivated ER recruits distinctive corepressor complexes. Cancer Res 66: 6370-6378, 2006.

118. Connolly RM, Li H, Jankowitz RC, Zhang Z, Rudek MA, Jeter SC, Slater SA, Powers P, Wolff AC, Fetting JH, et al: Combination epigenetic therapy in advanced breast cancer with 5-azacitidine and entinostat: A phase II National Cancer Institute/Stand up to cancer study. Clin Cancer Res 23: 2691-2701, 2017.

119. Anderberg C, Li H, Fredriksson L, Andrae J, Betsholtz C, Li X, Eriksson U and Pietras K: Paracrine signaling by platelet-derived growth factor-CC promotes tumor growth by recruitment of cancer-associated fibroblasts. Cancer Res 69: 369-378, 2009.

120. Roswall P, Bocci M, Bartoschek M, Li H, Kristiansen G, Jansson S, Lehn S, Sjölund J,ReidS, Larsson C, et al: Microenvironmental control of breast cancer subtype elicited through paracrine platelet-derived growth factor-CC signaling. Nat Med 24: 463-473, 2018.

121. Park JJH, Hsu G, Siden EG, Thorlund K and Mills EJ: An overview of precision oncology basket and umbrella trials for clinicians. CA Cancer J Clin 70: 125-137, 2020. International (CC BY-NC-ND 4.0) License. 